\title{
Analyse d'une activité de conception collaborative de ressources pour l'enseignement de la physique et la formation des professeurs
}

Le rôle de théories ou outils spécifiques

Analysis of the cooperative design of teaching resources for physics teachers:

some specific tools and theories

Laurent Veillard, Andrée Tiberghien et Jacques Vince

\section{(e) OpenEdition}

\section{Journals}

Édition électronique

URL : http://journals.openedition.org/activites/2627

DOI : 10.4000/activites.2627

ISSN : 1765-2723

Éditeur

ARPACT - Association Recherches et Pratiques sur les ACTivités

Référence électronique

Laurent Veillard, Andrée Tiberghien et Jacques Vince, « Analyse d'une activité de conception collaborative de ressources pour l'enseignement de la physique et la formation des professeurs », Activités [En ligne], 8-2 I octobre 2011, mis en ligne le 15 octobre 2011, consulté le 30 avril 2019. URL http://journals.openedition.org/activites/2627 ; DOI : 10.4000/activites.2627

\section{(c) (i) (2) $\Theta$}

Activités est mis à disposition selon les termes de la licence Creative Commons Attribution - Pas d'Utilisation Commerciale - Pas de Modification 4.0 International. 


\title{
Analyse d'une activité de conception collaborative de ressources pour l'enseignement de la physique et la formation des professeurs : le rôle de théories ou outils spécifiques
}

\author{
Laurent Veillard
}

laurent.veillard@univ-lyon2.fr

\section{Andrée Tiberghien}

andree.tiberghien@univ-lyon2.fr

\author{
Jacques Vince \\ jacques.vince@ens-lyon.fr
}

\begin{abstract}
Equipe COAST - UMR ICAR (Interactions Corpus Apprentissage Représentations)
ENS de Lyon, 15 Parvis Descartes, F 69007 Lyon
\end{abstract}

\begin{abstract}
Analysis of the cooperative design of teaching resources for physics teachers: some specific tools and theories. This work is situated in didactics of science, in a perspective of research-based design of teaching resources. In the first part, we present a specific theory (the theory of the two worlds) and the associated design tools drawn from more general theories on learning and epistemology in the field of physics, as well as from teachers' experience. This theory and the related tools constitute a theoretical basis for the practical choices involved in designing teaching sequences in physics and chemistry at high school level. In the second part, we analyse the collective activity of an R\&D group composed of one researcher and five teachers during a one-hour working session. The theoretical framework used is the theory of activity (Leontiev, 1979). The analysis focuses in particular on the role of the specific theory of two worlds and related tools in the group's work, and the outcomes in terms of practical decisions about the resulting teaching resources. Our findings show that this theory and the related tools work well for planning activities and learning situations, but they also show their limits when it comes to guiding teachers' work in the classroom. In the third part, we put forward some ideas for a complementary theory based on the Theory of Joint Action in Didactics that would form the theoretical foundation of the actions suggested to teachers for the design of teaching sequences.
\end{abstract}

KEYWORDS

Design of teaching resources, science education, physics teaching, analysis of activity, activity theory 


\section{Introduction}

Depuis plus d'une dizaine d'années, des chercheurs en didactique des sciences ${ }^{1}$ du laboratoire ICAR (Interactions Corpus Apprentissage Représentation, équipe COAST) collaborent avec plusieurs groupes d'enseignants d'une même discipline pour concevoir des ressources pour l'enseignement et la formation continue à partir de résultats de recherche et de l'expérience professionnelle des enseignants. Ces groupes de Recherche et Développement, appelés groupes SESAMES (Situations d'Enseignement Scientifique : Activités de Modélisation, d'Évaluation et de Simulation) regroupent des chercheurs (en général un ou deux) et des enseignants du secondaire (collège et lycée).

L'objectif de ces groupes est la conception de séquences d'enseignement conformes aux programmes nationaux, accompagnées de commentaires pour la mise en œuvre en classe et de vidéos d'élèves montrant certaines difficultés de compréhension. Ces séquences sont accessibles notamment par le biais d'un site internet (pegase.inrp.fr) ou de DVD conçus sur un thème plus spécifique. Les groupes conçoivent également des ressources pour la formation et des sessions de formation continue visant à aider les enseignants à mettre en œuvre des situations d'enseignement en s'appuyant sur les outils théoriques issus de la recherche. Une des modalités importantes pour atteindre cet objectif est de permettre aux enseignants de prendre conscience d'actions d'enseignement essentielles pour aider les élèves à comprendre les contenus d'enseignement.

Le type de conception développé ici relève d'une activité de recherche où l'on tente de prendre en compte la pratique professionnelle de ceux qui vont utiliser les ressources. Ainsi, les multiples choix faits lors de la conception sont fondés non seulement sur des recherches en didactique des sciences sur le fonctionnement du savoir et l'apprentissage des élèves, mais aussi sur l'expérience des enseignants (Design-Based-Research-Collective, 2003; Meheut \& Psillos, 2004). La stratégie développée dans les groupes SESAMES est de permettre une explicitation des contraintes, normes et pratiques enseignantes afin qu'elles puissent être prises en compte dans le processus de conception et devenir de possibles objets de débats en lien avec les recherches sur le savoir et l'apprentissage des élèves.

Notre hypothèse est que des instruments théoriques peuvent aider ce travail d'explicitation des choix. Celui-ci ne peut pas se faire à partir de théories générales telles que le constructivisme ou le socioconstructivisme qui orientent certes des choix d'apprentissage, mais ne peuvent fournir aucun modèle précis sur la progression d'un contenu d'enseignement et sa mise en ouvre concrète dans la classe. Il n'est donc pas surprenant qu'une même théorie générale conduise souvent les concepteurs à des choix de conceptions différentes (diSessa, 2006). Une telle stratégie de conception d'explicitation rend nécessaire des théories qui « are accountable to the activity of design. The theory must do real work » (Design-Based-Research-Collective, 2003, p. 10). De telles théories sont appelées spécifiques. Elles peuvent intégrer les expériences professionnelles des enseignants et plus généralement des normes et pratiques propres au métier. Elles doivent aussi pouvoir se traduire, pour une part, dans des outils sous forme de représentations externes qui soient directement opérationnels pour la conception et la mise en œuvre des ressources ${ }^{2}$ (Cobb, Confrey, diSessa, Lehrer, \& Schauble, 2003). De telles théories spécifiques doivent être beaucoup plus près des contenus et situations concrètes d'enseignement, par exemple dans notre cas l'enseignement de la physique au lycée.

1. Un travail similaire est mené en mathématiques, là aussi par un groupe R\&D composé d'un chercheur et de plusieurs professeurs. Les séquences produites partagent certaines hypothèses générales avec le groupe physique mais sont basées sur une approche théorique différente, issue des travaux en didactique des mathématiques. Les ressources produites sont en ligne sur le site PEGAME (http://pegame.inrp.fr/).

2. Cela rejoint aussi de nombreux travaux sur l'activité de conception collaborative, dans différents domaines, qui mettent en évidence l'importance des instruments mobilisés, notamment sous la forme de représentations externes, pour aider les concepteurs à créer un espace référentiel commun (Giboin, 2004), guider les inférences ou encore faciliter l'explicitation des choix (Darses, 2009). 
Dans cet article, notre objectif est d'étudier le rôle effectif que peuvent jouer des théories et outils de ce type dans une activité de conception de ressources pour l'enseignement de la physique. En effet, le travail mené au sein des groupes SESAMES depuis plusieurs années s'est accompagné d'un important travail réflexif sur le processus même de conception des séquences d'enseignement et des ressources de formation. Dans le cas de l'enseignement de la physique-chimie, plus particulièrement au lycée, il a permis d'aboutir à la construction d'une théorie spécifique et d'outils opératoires guidant le processus de conception. Dans la première partie de cet article, nous expliquons en quoi consistent cette théorie et ces outils et les caractéristiques des ressources produites. Puis, dans une deuxième partie, nous étudions leur opérationnalité et limites pour la conception de séquences d'enseignement de la physique en classe de seconde par un groupe R\&D composé d'un chercheur et plusieurs enseignants. Pour ce faire, une analyse de l'activité de conception collaborative est menée, à partir de la théorie de l'activité (Leontiev, 1979) et d'une typologie des savoirs mobilisés par les concepteurs. En conclusion, nous faisons quelques propositions pour enrichir les instruments à disposition des concepteurs afin qu'ils puissent davantage fonder les recommandations fournies aux enseignants utilisateurs.

Si ce travail se situe prioritairement en didactique des sciences, il nous semble qu'il rejoint des préoccupations plus générales portées par les chercheurs s'intéressant à l'analyse du travail enseignant, et sans doute plus largement aux activités de service (Mayen, 2005) et aux processus permettant sa transformation. Il s'agit en particulier de mieux comprendre comment des savoirs issus de l'expérience des praticiens et des travaux issus de la recherche (en l'occurrence ici plus particulièrement sur l'apprentissage et le fonctionnement des savoirs) peuvent être articulés pour aboutir à des pratiques enseignantes plus efficaces du point de vue de l'apprentissage des élèves. La question posée ici est plus particulièrement centrée sur les caractéristiques des espaces collaboratifs et des instruments d'aide à la conception qui peuvent favoriser ce type d'articulation.

\section{1.- Une théorie spécifique et des outils de conception}

Progressivement, un travail théorique mené à partir d'un retour réflexif sur l'activité de conception du groupe $\mathrm{R} \& \mathrm{D}$ en physique-chimie et sur son évolution sur une période d'une dizaine d'années, documenté par l'évolution des résultats et des concepts de la recherche, a permis de concevoir un cadre théorique pour la conception (Ruthven, Leach, Laborde, \& Tiberghien, 2009; Tiberghien, Vince, \& Gaidioz, 2009). Cette approche théorique repose sur deux hypothèses :

- Un cadre théorique pour la conception ne peut s'appuyer sur un seul type de théories générales (par exemple sur l'apprentissage ou bien sur l'épistémologie des connaissances enseignées) si l'on veut assurer la réalisation de ressources ainsi que l'explication et la communicabilité de leur processus de conception, dans la mesure où les situations d'enseignement sont des situations complexes non réductibles à un seul type de théories. Dans notre approche, nous mobilisons trois grands types de théories renvoyant aux trois pôles du triangle didactique : sur le fonctionnement ou l'épistémologie des connaissances dans la classe, sur l'apprentissage des élèves et sur l'activité ou l'action d'enseignement (cf. Figure 1).

- Le repérage et l'explicitation de plusieurs théories générales ("grand theories ») ne sont que la première étape d'un processus plus complexe de construction d'un cadre théorique pour la construction de ressources : en effet, il convient ensuite de passer à des constructions théoriques beaucoup plus spécifiques, qui permettent véritablement de fonder des choix de conception (« do real work ») et aboutir enfin à des outils utilisables par des concepteurs de ressources. 


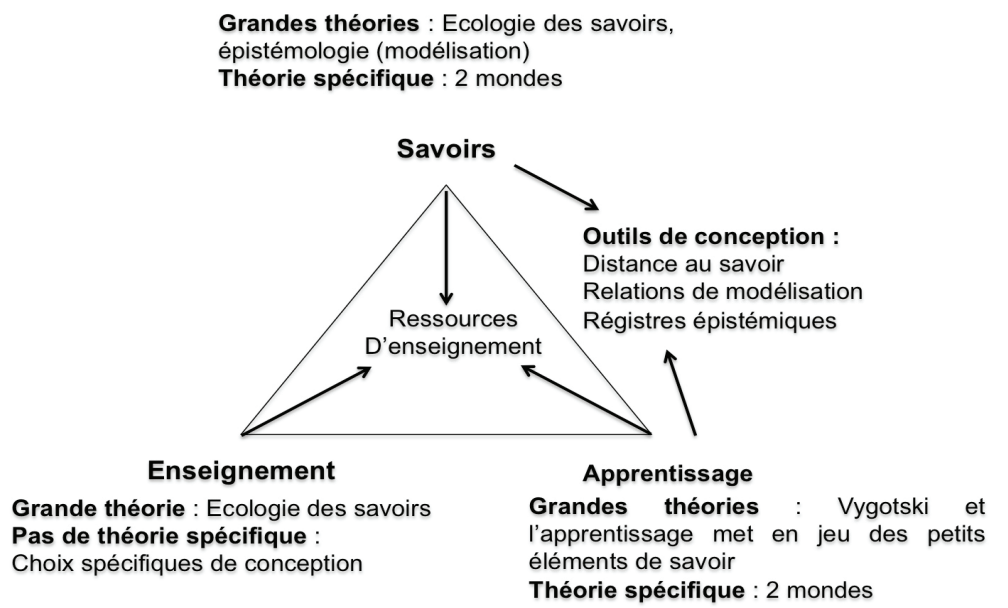

Figure 1 : Des grandes théories aux outils de conceptions

Figure 1: From main theories to design tools

\section{Les grandes théories sources de la théorie spécifique}

Le travail a été mené jusqu'ici dans une perspective ancrée en didactique des sciences. Du fait de l'orientation des recherches menées, il a conduit à développer une théorie spécifique plus fortement ancrée sur les pôles savoirs et apprentissage du système didactique, même si le pôle enseignement est un peu pris en compte. Cette théorie spécifique, nommée théorie des deux mondes, repose principalement sur trois sources théoriques générales (appelées grandes théories) : une approche anthropologique des savoirs; une approche épistémologique considérant la modélisation du monde physique comme une activité centrale en physique; le socio-constructivime Vygostkien. Les deux premières grandes théories portent sur le fonctionnement du savoir; la troisième sur l'apprentissage.

Le travail de Chevallard $(1991,1992)$ sur l'écologie des savoirs, d'abord en didactique des mathématiques, puis plus généralement sur les savoirs scolaires, a permis d'analyser les processus de transposition didactique des savoirs en jeu dans l'institution scolaire et de faire des distinctions entre les savoirs produits par les sphères scientifiques, les savoirs à enseigner formalisés dans les programmes, les manuels et les savoirs effectivement enseignés dans une classe particulière. Cette distinction est essentielle pour la conception des séquences SESAMES qui se situe clairement à l'articulation des savoirs à enseigner et des savoirs enseignés dans une classe. Les études menées sur la transposition didactique (Johsua \& Dupin, 1993), ainsi que des recherches menées dans une perspective ergonomique (Amigues, 2000) mettent en évidence l'importance du travail des enseignants pour interpréter les savoirs prescrits dans les programmes et les mettre en scène dans des situations de classes réelles afin que les élèves puissent vraiment se l'approprier. Partant de cette analyse, les séquences et ressources SESAMES sont élaborées avec une double contrainte : respecter le programme, condition essentielle pour qu'elles soient crédibles vis-à-vis des enseignants et légitimes vis-à-vis de l'institution scolaire; aider les enseignants à réduire l'écart entre les prescriptions officielles et le savoir enseigné, en tenant compte au maximum des conditions concrètes d'enseignement et en fournissant, par exemple, des documents écrits qui sont directement utilisables par les élèves, auxquels sont attachés des commentaires de mise en œuvre à destination des enseignants.

Le travail de conception s'appuie également sur une épistémologie qui place l'activité de modélisation du monde matériel au cœur de la physique. Basée sur les propositions de plusieurs épistémologues (Bachelard, 1979; Bunge, 1973; Giere, 1988; Hacking, 1983/2005), cette approche se caractérise par les points suivants. Tout d'abord, nous considérons que les activités d'observation et d'expérimentation sont loin d'être toujours guidées par la théorie physique. Plusieurs exemples 
dans l'histoire de cette discipline montrent que des observations n'ont pas été initiées par des hypothèses théoriques, mais par des qualités de curiosité, de réflexivité des scientifiques. Elles ont de fait précédé toute formulation théorique. De ce point de vue, les moments d'observation et d'expérience sont des activités fondamentales dans toute formation scientifique et ils ne peuvent être remplacés par des descriptions écrites (Hacking, 1983/2005). Une deuxième idée forte porte sur le rôle de la modélisation comme lien entre les propositions théoriques et les expériences. Nous suivons sur ce point Bachelard (1979) et Hacking (1983/2005) qui considèrent que l'articulation entre théories et expériences ne peut se faire directement, mais nécessite l'élaboration de modèles. La construction de modèles renvoie à deux processus : l'un partant de la théorie, pour la rendre plus concrète; l'autre partant de l'expérience pour la rendre plus abstraite. Nous appelons modélisation ce double processus et modèle son résultat. De ce fait, un modèle est un intermédiaire entre la théorie et l'expérience et possède en quelque sorte deux facettes.

La troisième grande théorie mobilisée porte sur l'apprentissage et le considère d'un point de vue socioconstructiviste. Ce point de vue nous conduit, d'une part à accorder un rôle fondamental aux connaissances préalables des élèves dans le processus d'apprentissage, en particulier celles construites dans l'expérience de la vie quotidienne et d'autre part à mettre en avant le rôle des situations d'enseignement comme vecteur social des apprentissages individuels (Vygotski, 1934/1997).

Des recherches sur les trajectoires individuelles d'apprentissage en classe de sciences (Givry, 2003; Küçüközer, 2005 ; Psillos \& Kariotoglou, 1999) ont mis en évidence que, la plupart du temps, elles ne suivent pas l'ordre d'introduction des savoirs par l'enseignant, ni même une décomposition logique du savoir disciplinaire. Lorsque l'étude est menée à différentes échelles d'analyse (Tiberghien \& Malkoun, 2007), on s'aperçoit que l'apprentissage peut consister à mettre en lien des éléments du savoir enseigné avec d'autres connaissances préalables, en particulier des théories naïves ou des savoirs acquis lors d'un enseignement précédent. Nous retenons l'idée que la compréhension d'un concept ou d'une notion en physique nécessite d'établir de nouvelles relations entre des éléments de savoirs qui peuvent être très petits. Ces relations peuvent être différentes de celles en jeu dans le savoir enseigné et les élèves peuvent acquérir des éléments partiels de ce savoir enseigné sans une compréhension conceptuelle complète de celui-ci. Nous en tirons la conclusion qu'un enseignement doit permettre aux élèves d'améliorer leur compréhension de nouveaux éléments de connaissance, qui peuvent être parfois relativement petits, en leur donnant la possibilité de réutiliser ceux-ci dans des activités successives. Ces activités doivent s'appuyer sur des temps d'échanges importants dans la classe entre les élèves et avec l'enseignant, afin de permettre une formulation fine des idées des uns et des autres et stimuler des processus d'évolution des significations sur le plan intersubjectif, préalable nécessaire à leur intériorisation individuelle (Vygotski, 1934/1997)

\section{La théorie spécifique des deux mondes et les outils associés}

À partir des trois grandes théories décrites ci-dessus, la théorie spécifique des deux mondes a été élaborée pour guider la conception de ressources d'enseignement de la physique au niveau secondaire ${ }^{3}$ (lycée). Nous ne présentons ici que les grandes lignes de cette théorie et nous renvoyons à un autre article pour une présentation plus détaillée (Tiberghien et al., 2009).

La théorie repose sur deux hypothèses principales :

1) L'activité centrale en physique est une activité qui consiste à faire le lien entre deux niveaux de connaissance ${ }^{4}$ qui renvoient à deux « mondes » différents : d'une part celui des constructions théoriques et d'autre part celui du champ expérimental des objets et événements. Cette mise en lien

3. A ce niveau, il n'y a pas besoin d'une instrumentation trop complexe car, soit les phénomènes sont directement observables (ex : pierre attachée à un élastique en mécanique), soit ils sont appréhendables par des appareils simples (ex : voltmètre, générateur en électricité) ou par le biais de simulateurs quand il s'agit de physique microscopique (ex : simulateur de mouvement des particules dans le cas du son). Les objets et les évènements sont donc facilement identifiables.

4. Niveau n'implique ici aucune idée de hiérarchie. 
nécessite une activité de modélisation importante qui consiste, d'un côté à sélectionner et traiter les éléments théoriques correspondants aux phénomènes et objets étudiés; de l'autre à sélectionner des objets et événements, à les décrire avec les mots de la physique et/ou à procéder à des expériences mesurables sur ces événements. Initialement, la théorie distinguait trois niveaux : théories (ayant une fonction explicative), modèles (ayant une fonction descriptive et interprétative), objets et événements. Mais les enseignants participant aux groupes de conception ont argué du fait que la distinction entre les différentes fonctions des théories et modèles serait trop subtile pour les élèves et les enseignants ne participant pas à la conception, essentiellement parce que la nuance entre théorie et modèle est assez peu visible au niveau de l'enseignement secondaire (Coince, Miguet, Perrey, Rondepierre, Tiberghien, \& Vince, 2008). Nous avons donc rapidement décidé de réduire notre modèle théorique à deux mondes, qui deviennent explicites pour les élèves et structurent leur activité. Le monde des « théories et modèles » renvoie donc aux concepts généraux et aux constructions abstraites plus locales permettant d'étudier un type d'objets et d'événements; le monde des objets et des événements renvoie à ce qui est accessible par la perception de façon directe (observation) et/ou par le biais d'instruments de mesure.

2) Les processus cognitifs en jeu dans la vie courante pour la compréhension du monde matériel peuvent également être appréhendés comme des processus de modélisation qui mettent en lien des ensembles de théories plus ou moins naïves et des objets et événements (Tiberghien, 2000). La vie quotidienne conduit ainsi d'un côté à des observations, voire des mesures (par exemple la température ambiante ou celle d'un four) et de l'autre à avancer des idées explicatives que l'on peut rattacher au monde des théories et modèles. La théorie spécifique des deux mondes peut donc être utilisée à la fois pour analyser les savoirs de la physique et les connaissances issues de la vie quotidienne. C'est d'ailleurs son grand intérêt puisque l'on a vu que l'apprentissage en classe avait de fortes chances de mettre en jeu non seulement des savoirs transposés de la physique, mais aussi ces conceptions quotidiennes. La figure 2 ci-dessous montre toutes les relations potentielles entre les niveaux et catégories de connaissances en jeu dans les activités d'enseignement de la physique en classe. Cette théorie spécifique permet d'être beaucoup plus attentif à ces différents savoirs et aux relations que les élèves peuvent et doivent en général construire entre différents niveaux et types de connaissances. En particulier, les mots utilisés peuvent être spécifiques à la physique, mais d'autres sont aussi utilisés dans la vie quotidienne avec un sens identique ou différent. Par exemple, «énergie », « force » ou encore «puissance » sont utilisées avec un sens différent de celui de la vie quotidienne. La théorie rend plus attentif à ces différences de sens, en poussant à expliciter les contextes d'utilisation des termes (Gaidioz, Vince, \& Tiberghien, 2004).

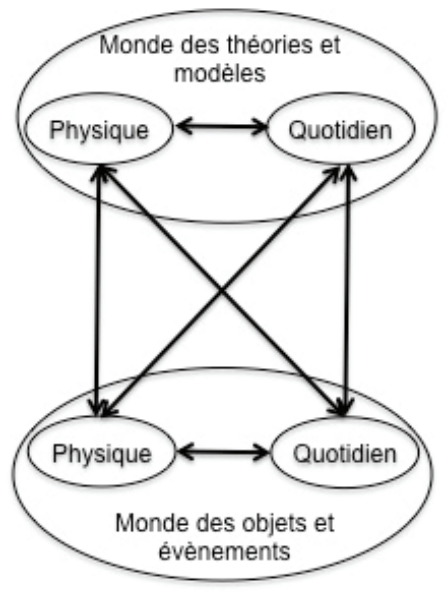

Figure 2 : Représentation de la théorie spécifique des deux mondes

Figure 2: Representation of the specific theory of two worlds 
Cependant, la construction de cette théorie spécifique ne s'est pas révélée suffisante pour assurer un guidage efficace du processus de conception au sein des groupes R\&D. À partir de cette théorie, nous avons donc décidé de concevoir trois outils plus opérationnels et complémentaires à la théorie spécifique des deux mondes : la distance entre types de savoirs; les relations de modélisation et les registres sémiotiques.

Le premier outil se présente sous forme d'un tableau (cf. Tableau 1 ci-dessous pour un exemple) à double entrée, et permet aux concepteurs de faire des hypothèses sur les différences et les distances entre les savoirs à enseigner (programmes) et les connaissances des élèves du point de vue du processus de modélisation en jeu.

\begin{tabular}{|c|c|c|c|c|}
\hline & \multicolumn{2}{|c|}{ déjà connu* } & \multicolumn{2}{|c|}{ À construire } \\
\hline & Physique-chimie & Quotidien & Physique-chimie & Quotidien \\
\hline $\begin{array}{c}\text { Théories et } \\
\text { modèles }\end{array}$ & $\begin{array}{l}\text { - Relation algébrique } \\
\text { entre période et } \\
\text { fréquence. } \\
\text { - Unités de fréquence et } \\
\text { période. } \\
\text { - Distinction valeur } \\
\text { maximale d'une } \\
\text { tension/période d'une } \\
\text { tension. }\end{array}$ & $\begin{array}{l}\text { - Une période est } \\
\text { une durée. }\end{array}$ & $\begin{array}{l}\text { - Signification des phénomènes } \\
\text { émission, réception, absorption, } \\
\text { réflexion, transmission. } \\
\text { - Les ondes sont caractérisées par } \\
\text { leur fréquence. } \\
\text { - Les domaines de fréquences } \\
\text { des sons et des ondes } \\
\text { électromagnétiques utilisés dans } \\
\text { les applications médicales ne } \\
\text { sont pas du tout les mêmes. }\end{array}$ & \\
\hline $\begin{array}{c}\text { Relations } \\
\text { Théorie/ } \\
\text { modèle } \\
- \\
\text { Objets/évé- } \\
\text { nements }\end{array}$ & $\begin{array}{l}\text { - Le son est une onde. } \\
\text { - Représentation } \\
\text { graphique d'un } \\
\text { phénomène périodique } \\
\text { en fonction du temps } \\
\text { repérage de la période. }\end{array}$ & \begin{tabular}{|l} 
- Une onde ne \\
traverse pas les \\
matériaux solides. \\
- Un ultrason est un \\
son qu'on n'entend \\
pas. \\
- Le son se déplace \\
beaucoup moins \\
vite que la lumière.
\end{tabular} & $\begin{array}{l}\text { - Description d'une situation en } \\
\text { termes de chaîne sonore. } \\
\text { - Valeur de la vitesse du son dans } \\
\text { l'air. } \\
\text { - Valeur de la vitesse de la lumière } \\
\text { dans l'air ou dans le vide. } \\
\text { - Des ultrasons sont des sons de } \\
\text { fréquence supérieure à } 20 \mathrm{kHz} \text {. } \\
\text { - Exploiter un dispositif pour } \\
\text { étudier les phénomènes } \\
\text { de réflexion, absorption, } \\
\text { transmission. }\end{array}$ & $\begin{array}{l}\text { - Principe de } \\
\text { l'écographie et de la } \\
\text { radiographie. }\end{array}$ \\
\hline $\begin{array}{c}\text { Objets et } \\
\text { événements }\end{array}$ & $\begin{array}{l}\text { - La signification du } \\
\text { mot fréquence dans la } \\
\text { vie courante n'est pas } \\
\text { toujours la même qu'en } \\
\text { physique (idem pour } \\
\text { période). }\end{array}$ & $\begin{array}{l}\text { - Caractère plus ou } \\
\text { moins fréquent } \\
\text { d'un événement. } \\
\text { - Phénomène de } \\
\text { l'écho. } \\
\text { - Échographie et } \\
\text { radiographie sont } \\
\text { des techniques } \\
\text { d'imagerie } \\
\text { médicales. }\end{array}$ & $\begin{array}{l}\text { - Échographie et radiographie } \\
\text { reposent sur des phénomènes } \\
\text { physiques différents et sur des } \\
\text { ondes de natures différentes. }\end{array}$ & $\begin{array}{l}\text { - On utilise l'une ou } \\
\text { l'autre des techniques } \\
\text { d'imagerie en fonction } \\
\text { des propriétés } \\
\text { de ce qu'on veut } \\
\text { «observer» vis-à-vis } \\
\text { des sons ou des ondes } \\
\text { électromagnétiques. }\end{array}$ \\
\hline
\end{tabular}

* Dans le cas de l'enseignement de la physique chimie, on a ici supposé connu ce qui a été déjà enseigné. Mais cela ne signifie pas nécessairement que les élèves ont compris conceptuellement ces savoirs.

Tableau 1 : Exemple de mise en œuvre de l'outil 1 sur le sous-thème « Diagnostic médical » du programme de seconde

Table 1: Example of implementing the tool 1 on the sub-theme of "Medical diagnosis".

Le second outil permet une explicitation des types de relations auxquels l'enseignement doit conduire les élèves, qui peuvent être de quatre types : 1) Relations entre objets et événements ;2) Relations entre objets et événements et théories/modèles; 3) Relations d'éléments théoriques/modèles à des objets et événements; 4) Relations au sein du monde des théories/modèles. Les concepteurs disposent ainsi d'un outil qui les aide à rendre plus explicites, pour chaque séquence d'enseignement, les objets et événements, les éléments théoriques en jeu et les types de relations de modélisation à construire dans les activités confiées aux élèves. Il a conduit notamment à élaborer un élément 
essentiel fourni aux élèves lors des activités réalisées : le texte du modèle. Nous y reviendrons dans la partie suivante de l'article.

Le troisième outil (registres sémiotiques) n'est pas directement tiré de la théorie spécifique des deux mondes, mais de travaux sur les registres sémiotiques (par exemple Duval, 1995). Nous reprenons ainsi à notre compte l'hypothèse que la construction de la signification d'un concept passe par la mobilisation de différents registres sémiotiques exprimant ce concept et leur mise en lien : langage naturel, schéma, tableau de mesure, etc. Ainsi dans notre cas, dans chacun des mondes de la théorie des deux mondes, il y a langage et sémioticité des objets. Cet outil vise à rendre les concepteurs attentifs à ce que les élèves recourent à différents registres sémiotiques et à analyser les éventuelles difficultés pour les mettre en lien, le langage naturel jouant un rôle essentiel pour établir ces relations, mais pouvant conduire à des interférences de sens comme déjà mentionné ci-dessus.

\section{Processus de conception générale et caractéristiques des ressources produites}

La théorie spécifique et les outils complémentaires décrits ci-dessus sont mobilisés au sein des groupes R\&D depuis environ 10 ans. Ils ont aidé à la conception de plusieurs séquences d'enseignement de la physique et de la chimie au lycée (Gaidioz \& Tiberghien, 2003; Gaidioz et al., 2004; Le Marechal, Barbe, Roux, Jean-Maraie, Roue, \& Vincent, 2004). La méthodologie de travail générale est la suivante. Le groupe composé du (ou des) chercheur(s) et des enseignants se retrouve chaque semaine ou tous les 15 jours pour des séances de travail qui durent trois heures. Ils travaillent selon un processus en quatre étapes, s'échelonnant sur deux années : pendant la première année, après une première analyse des savoirs en jeu grâce aux outils de conception (étape 1), une première version des séquences et des documents associés est élaborée (étape 2); ceux-ci sont ensuite testés par les enseignants du groupe dans leur classe, quand cela est possible très rapidement (étape 3); puis lors de la deuxième année, un retour critique est opéré sur ces séquences à partir des expériences de mise en œuvre des enseignants-concepteurs dans des classes différentes (étape 4). Dans certains cas, des recherches axées sur l'activité et les apprentissages des élèves au cours de ces séquences sont prises en compte. Enfin, il est courant que les ressources soient encore largement modifiées les années suivantes après retour d'expérimentation d'enseignants (en particulier ceux exerçant dans les mêmes établissements que les membres du groupe $\mathrm{R} \& \mathrm{D}$ ) ou suite aux sessions de formations encadrées par le groupe.

Les séquences d'enseignement élaborées présentent des caractéristiques qui les distinguent de l'enseignement plus usuel de la physique au lycée. Tout d'abord, il n'y pas de cours pour présenter les connaissances. Les nouveaux savoirs sont introduits par des activités, rapidement présentées par l'enseignant et que les élèves conduisent ensuite en petits groupes. Elles peuvent être de natures variables, plus ou moins longues, plus ou moins ouvertes, recourir ou non à la manipulation. Elles favorisent une activité autonome des élèves et une première construction de nouveaux savoirs par la discussion, la prévision/vérification, la manipulation, la lecture de documents et l'écriture d'interprétations. Les élèves disposent d'un document qui comprend les consignes pour la réalisation des activités et dans beaucoup de cas, un modèle théorique permettant d'étudier les situations proposées (voir exemple en annexe 2), conformément à la théorie spécifique des deux mondes. Ce modèle écrit leur donne la responsabilité d'évaluer leurs propositions en référence à la théorie de la physique et ainsi d'établir des relations entre les deux mondes. Il constitue une référence théorique commune dans la classe et permet à l'enseignant de dépersonnaliser le savoir de la physique. Après le travail en petits groupes, une deuxième phase, cruciale, implique toute la classe dans une discussion à propos des procédures et des propositions des élèves. À la fin de cette phase, l'enseignant indique quelles sont les connaissances pertinentes du point de vue de la physique et les institutionnalise. Cette structuration de l'enseignement en activités modifie évidemment l'articulation traditionnelle entre les cours et les TP. La séance de TP en demi-classe n'est plus à côté du cours (comme vérification d'un résultat de cours ou introduction expérimentale à un nouveau sujet par exemple), mais les deux types de séances s'enchaînent sans qu'il y ait forcément de rupture quant à la nature des connaissances à 
construire, et les élèves travaillent en petits groupes aussi bien en classe entière qu'en demi-classe.

Les documents élèves sont accompagnés de documents à destination des enseignants pour aider ces derniers à mettre en œuvre les séquences. Chaque activité donne lieu à cinq rubriques de commentaires pour l'enseignement :

- but de l'activité (insertion dans la progression du savoir, objectifs explicites pour l'élève);

- informations sur la préparation de l'activité (durée, nécessité éventuelle d'un effectif réduit, matériel nécessaire et informations sur l'expérimentation);

- informations sur le savoir (analyse du savoir en jeu à partir des théories spécifiques, justification des choix faits à partir des outils de conception, compléments didactiques ou épistémologiques);

- informations sur le comportement des élèves (idées initiales risquant d'émerger, difficultés principales, rubrique parfois illustrée par des vidéos d'élèves en train de faire l'activité);

- corrigé de l'activité (réponses attendues).

En plus de ces documents écrits, des formations sont proposées aussi bien pour les enseignants (formations académiques) que pour les formateurs (formations nationales). Pour les formations d'enseignants, les séquences produites sont souvent le point de départ de la formation, mais les choix faits sont justifiés par les outils de conception qui tendent à devenir décontextualisés pour appropriation par les enseignants. Pour les formations de formateurs, le processus est inverse : les séquences d'enseignement servent à exemplifier la prise en compte des théories et des outils de conceptions. On retrouve cette articulation entre séquences et outils de conception sur le site pegase.inrp.fr, structuré en deux rubriques, Enseigner et Se former.

\section{2.- Opérationnalité et limites de la théorie spécifique et des outils dans le processus de conception de ressources}

Dans cette seconde partie, nous allons nous pencher sur l'activité de conception de ces séquences et des ressources associées. Notre objectif est d'analyser le rôle que jouent effectivement la théorie spécifique et les outils de conception dans le travail du groupe R\&D, en particulier si ces instruments (au sens Vytgotskien) parviennent à expliciter et guider les choix des concepteurs. Il s'agit aussi d'en éprouver les limites et de mieux caractériser les moments de l'activité du groupe au cours desquels ils ne fonctionnent plus (au sens où ils ne peuvent plus assurer de fonction). En effet, deux pôles ont été privilégiés pour la construction de la théorie spécifique des deux mondes. Le manque d'appui sur le pôle enseignement conduit-il à passer sous silence ou être plus flou quant à certaines actions enseignantes qui doivent pourtant être prises en compte dans une logique de transférabilité et de mise en œuvre des séquences d'enseignement? Notre hypothèse, étayée sur les retours des différents groupes SESAMES, mais à mettre à l'épreuve à partir de l'étude de cas réalisée ici, est que cette dimension est sans doute trop faiblement contrôlée par des choix théoriques plus précis et qu'une analyse de l'activité de conception peut permettre de trouver des pistes pour mieux documenter la construction des séquences et guider leur mise en œuvre.

\section{Approche théorique et méthodologie pour l'analyse des séances de conception}

Pour analyser l'activité de conception du groupe $\mathrm{R} \& \mathrm{D}$, nous reprenons et développons une approche théorique et méthodologique initiée dans le cadre d'un précédent travail de recherche portant sur l'appropriation de séquences SESAMES par un groupe de trois enseignants (Jeannin, Veillard, \& Tiberghien, 2010). Cette approche est fondée sur la théorie de l'activité développée par Leontiev (1979) dans le prolongement de la psychologie de Vygotski (1978)

Nous considérons que les enseignants et le chercheur qui participent à ce groupe sont engagés dans une activité dont ils sont le sujet collectif et dont le motif est d'aider les professeurs à enseigner la 
physique dans le sens d'une amélioration des apprentissages des élèves. Pour cela, les acteurs travaillent sur des objets culturels particuliers qui sont des séquences d'enseignement. Les résultats produits par l'activité collective sont les séquences labélisées SESAMES, ainsi que les documents (documents "profs" pour aider l'enseignant à s'approprier et à mettre en œuvre les séquences) et les formations pour les enseignants. L'activité se réalise par le biais d'actions collectives avec des composantes verbales (échanges langagiers entre les acteurs autour de la table) et opératoires (opérations symboliques ou matérielles entraînant des modifications des séquences). Ces actions portent sur des objets et sont initiées et orientées par les buts, des préoccupations, des propositions de chaque participant. Une action peut s'interrompre et reprendre plus tard. Elle peut aussi ne pas déboucher sur un résultat (choix de conception en particulier). Cette activité n'est pas directe, mais médiée par des outils (artefacts techniques et instruments culturels). Les instruments culturels, qui peuvent être des savoirs ou connaissances ${ }^{5}$, de différents types, nous intéressent ici plus particulièrement ${ }^{6}$. Nous avons élaboré une typologie de ces différents types d'instruments à partir de travaux sur les connaissances des enseignants (Doudin, Pfulg, Martin, \& Moreau, 2001; Shulman, 1986; Woolfolk Hoy, Davis, \& Pape, 2006) que nous avons adaptés au contexte du groupe R\&D de conception :

- I1 : le programme et les instructions officielles;

- I2 : des savoirs de la physique;

- I3 : des savoirs de la vie quotidienne;

\section{- I4 : la théorie spécifique des deux mondes et les outils de conception;}

- I5 : des ressources déjà produites par le groupe (séquences ou « docs profs » écrits avant la séance par un des concepteurs ou conçus les années précédentes);

- I6 : des savoirs didactiques (recherches menées sur la mise en œuvre de ces séquences, recherches sur les idées initiales des élèves ou d'autres recherches) ou d'autres disciplines des sciences humaines;

- I7 : des connaissances, parfois formalisées sous forme écrite ou simplement verbalisées lors de la séance, sur les situations concrètes d'enseignement (ex. : contraintes temporelles, organisationnelles, institutionnelles, réaction et difficultés des élèves, etc.); ces connaissances peuvent être issues de l'expérience d'enseignement des enseignants ou de l'expérience de pairs;

- I8 : d'autres instruments tels que des manuels scolaires, des ouvrages, des outils techniques, etc.

La théorie spécifique des deux mondes et les outils associés (I4) constituent un type spécifique d'instrument en termes d'impact dans le travail collaboratif du groupe par rapport à d'autres types.

Chacun de ces instruments peut jouer tour à tour la fonction de ressource ou de contrainte pour/sur l'activité. Ainsi, il est clair que les programmes constituent des prescriptions sur le savoir à enseigner, formulées en termes de contenus et de compétences attendues, qui jouent comme des contraintes fortes chez les enseignants (Amigues, 2000), qu'il faut respecter si l'on souhaite que les séquences soient reprises par beaucoup de praticiens. Mais ils jouent aussi simultanément le rôle de ressources pour les concepteurs, lorsque ceux-ci s'appuient sur ces textes pour analyser la structure conceptuelle des savoirs en jeu et en définir les limites (quel concept supplémentaire ne figurant pas au programme doit être nécessairement traité, quel concept ne doit pas l'être?). Les programmes mobilisent dans ce cas également les savoirs de la physique, et très souvent la théorie spécifique des deux mondes.

Sur le plan méthodologique, nous avons procédé à l'enregistrement vidéo de trois réunions d'une durée variable de $1 \mathrm{~h}$ à $2 \mathrm{~h} 30$ environ, regroupant un chercheur et cinq enseignants disposés de part et d'autre d'une table et travaillant avec un micro-ordinateur relié à un vidéo-projecteur. Le dispositif de captation était relativement simple avec une caméra prenant les acteurs et l'écran et 1 micro d'ambiance posé sur la table (cf. Figure 3).

5. Savoir et connaissances sont ici considérés comme synonymes.

6. Nous ne prendrons pas en compte les artefacts techniques tels que les micro-ordinateurs, le vidéo projecteur ou encore le site internet pégase qui jouent un rôle indéniable dans le processus de conception mais qui ne font pas l'objet de notre analyse. 


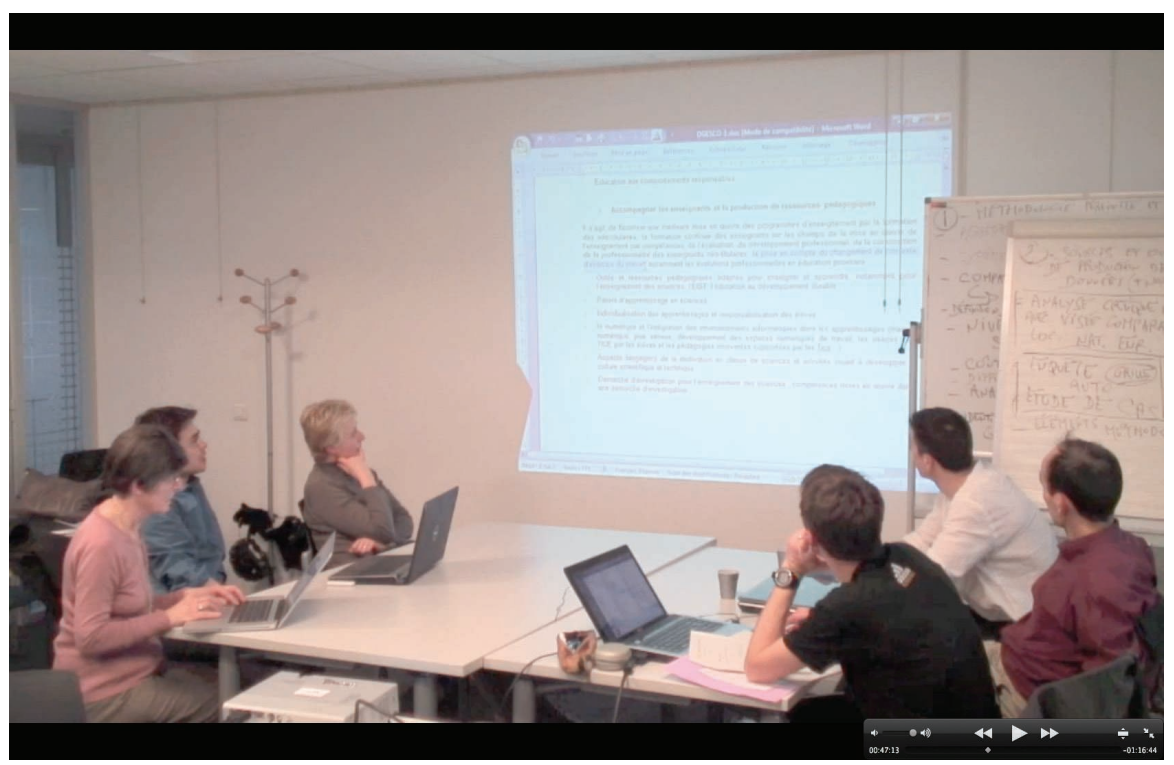

Figure 3 : Configuration de l'espace de travail

Figure 3: Configuration of the workspace

Pour cet article, nous avons fait le choix de nous centrer sur l'analyse de la première séance enregistrée qui dure une heure. La transcription complète des échanges a été réalisée à l'aide du logiciel Transana qui nous a également servi pour le découpage en différentes actions. Pour ce faire, les échanges entre les participants ont été découpés en fonction des objets sur lesquels ils portaient et des buts visés par le groupe. Ainsi, l'action A3 « Simplifier un tableau trop compliqué » est initiée par une préoccupation du chercheur qui n'a pas compris le fonctionnement du tableau (cf. Extrait 1 ci-dessous). Les échanges qui suivent visent d'abord l'explicitation du principe de sa construction, puis sa modification, après que les participants se soient mis d'accord sur sa trop grande complexité.

Puis nous avons mené une analyse qualitative des instruments mobilisés pour chaque action, en lien avec les décisions prises par rapport à des modifications de la séquence ou à des recommandations faites aux enseignants pour sa mise en œuvre (cf. Tableau synoptique en annexe 3). Nous avons ainsi pu identifier quelques extraits plus intéressants du point de vue du rôle que pouvait y jouer la théorie spécifique des deux mondes et les outils de conception associés. Ces extraits ont fait l'objet d'une analyse plus détaillée.

\section{Position des extraits analysés dans le processus de conception et la séance}

Avant de rendre compte de nos analyses, il est nécessaire de dire quelques mots sur la situation des extraits analysés dans l'ensemble du processus de conception, puis dans la réunion.

Au cours de cette réunion, le groupe travaille principalement sur une séquence d'enseignement de la physique en seconde portant sur les ondes sonores et électromagnétiques, puis en fin de réunion plus brièvement sur une autre séquence en mécanique. ${ }^{7}$

Ce travail de conception a lieu dans le cadre de la mise en œuvre d'un nouveau programme en classe de seconde. Les enseignants concepteurs ont pour la plupart déjà travaillé sur les savoirs de la discipline en jeu dans le contexte du groupe $\mathrm{R} \& \mathrm{D}^{8}$. Cependant la nouveauté réside essentiellement dans

7. La première heure de la séance n'a pas été transcrite car lors de cette première partie de la réunion, le groupe travaille sur une demande de moyens auprès de l'IFE (ex INRP) pour assurer son fonctionnement l'année suivante. Cela explique que la transcription démarre avec un timecode à 1:01:50.

8. Il s'agit de cinq enseignants de physique dont deux exercent dans le même établissement. Quatre d'entre eux (P1, P2, P3, P5) ont une expérience de fonctionnement dans le groupe de plus de cinq ans. Pour P4, il s'agit de sa première année et il n'a que quelques mois comme expérience de concepteur. Il a par contre déjà mis en œuvre des séances 
la prise en compte d'une nouvelle prescription, à savoir la présentation des savoirs par thèmes : par exemple les ondes doivent être traitées dans le sous-thème «diagnostic médical » du thème santé pour les ondes; la mécanique est traitée dans le thème « sport », etc. L'objectif du groupe est d'articuler une analyse du savoir et des connaissances initiales des élèves sur le sujet préalable à la séance étudiée (et au cours de laquelle la théorie spécifique a fonctionné) avec une prescription institutionnelle, gage incontournable d'une utilisation potentielle par les pairs des ressources produites.

$\mathrm{Au}$ début de la réunion et pendant une grande partie de celle-ci, les concepteurs discutent d'une proposition de séquence sur les ondes électromagnétiques écrite par P3 et P4 (projetée via le vidéoprojecteur depuis l'ordinateur portable de P3). Lors de la réunion précédente, le groupe a conduit une analyse du savoir en jeu dans cette partie du programme à l'aide de la théorie spécifique et des outils. Il en est sorti non seulement une structure conceptuelle de la séquence, mais aussi des choix arrêtés quant à l'articulation entre savoirs de la physique et applications technologiques présentées dans le cadre de l'approche thématique (échographie et radiographie, etc.). Les concepteurs ont décidé de reprendre quelques activités d'une ancienne séquence et de s'inspirer de ce que font deux des enseignants ( $\mathrm{P} 3$ et $\mathrm{P} 4)$ dans le même établissement.

En ce qui concerne la séquence en mécanique, la situation est différente : une séquence existe, parfaitement conforme au contenu du nouveau programme, mais sans le lien avec la thématique du sport. Cette séquence a été la plus travaillée par le groupe depuis 10 ans. Les membres du groupe savent qu'elle est utilisée par de nombreux enseignants et qu'elle est robuste au sens où elle s'adapte bien aux différents contrats didactiques mis en place par les enseignants. La question principale qui se pose au groupe est donc de savoir si on l'adapte au thème sport et si oui comment la réduire éventuellement au regard des énormes contraintes de temps que les enseignants perçoivent au sujet du nouveau programme.

On trouvera en annexe un tableau synoptique de la chronologie des actions lors de cette séance, avec une indication sur les instruments mobilisés lors de chacune d'elle. Les extraits qui vont être analysés ci-dessous correspondant aux actions numéro A1a/b, A3, A4a/b, A11.

\section{Deux actions aboutissant à des décisions dans le processus de conception}

Nous allons d'abord nous pencher sur deux moments de la séance où les concepteurs s'appuient sur les principes de la théorie spécifique qui instrumentent l'échange et permettent d'aboutir à des décisions effectives dans le processus de conception.

Le premier extrait est relatif à l'action A3 dans le tableau synoptique (« simplifier un tableau trop compliqué »). Comme on l'a déjà dit précédemment, l'échange est initié dans ce cas par le chercheur (C) qui ne comprend pas une partie du tableau final figurant dans l'activité 5 du chapitre sur les ondes (cf. extrait 1). Ce tableau (cf. Tableau 2) vient après des questions portant respectivement sur la technique de l'échographie qui met en jeu la réflexion d'ondes, et la technique de la radiographie qui s'appuie sur le phénomène de transmission. Le tableau vise à bien différencier ces deux phénomènes physiques en fonction des caractéristiques des deux types d'objets techniques (émetteur et récepteur placés du même côté ou de part et d'autre).

Extrait1 - 1:05:28

- C : et puis moi j'ai pas très bien compris heu ce tableau-là cocher les bonnes réponses dans la colonne (montre la colonne de droite du tableau)

- P3 : alors certains élèves ont pas très bien compris non plus d'ailleurs (rires)

- C: j'ai pas compris [...] je vois ce que tu veux dire, mais tel quel heu qu'est-ce que analyse de l'onde réfléchie alors tu analyses l'onde réfléchie du même côtél

SESAMES dans sa classe depuis quelques années, en proposant parfois des retours critiques avant même sa participation au groupe. Le chercheur est l'initiateur de ce groupe il y a plus de dix ans. 


\begin{tabular}{|c|c|c|c|}
\cline { 2 - 4 } \multicolumn{1}{c|}{} & \multicolumn{2}{c|}{ Émetteur/capteur placés } & \multicolumn{1}{c}{ Exemple } \\
\cline { 2 - 4 } \multicolumn{1}{c|}{} & $\begin{array}{c}\text { du même côté du } \\
\text { corps à analyser }\end{array}$ & $\begin{array}{c}\text { de part et d'autre } \\
\text { du corps à analyser }\end{array}$ & échographie $\square$ oui $\square$ non \\
Analyse de l'onde réfléchie & & & radiologie $\square$ oui $\square$ non \\
\hline Analyse de l'onde transmise & & & échographie $\square$ oui $\square$ non \\
radiologie $\square$ oui $\square$ non
\end{tabular}

Tableau 2 : Initial

Table 2: Initial

L'enseignant qui a conçu le tableau (P4) chez lui avant cette séance explique qu'il est d'abord parti des deux types de phénomènes (onde réfléchie/transmise) pour ensuite accoler les exemples d'applications technologiques. Mais il reconnaît la trop grande complexité du tableau. La réflexion s'engage pour trouver une présentation plus simple. P3 propose de supprimer la colonne de droite, ce que valident plusieurs membres du groupe. Puis P5 suggère de partir des deux applications technologiques (en ligne) plutôt que des phénomènes physiques, ce qui est immédiatement acté. Mais ces modifications du tableau ont comme conséquence que celui-ci ne fait plus apparaître les phénomènes physiques, mais seulement les dispositions respectives des émetteurs et récepteurs. P5 s'en aperçoit le premier (cf. Extrait 2).

Extrait 2 - 1:07:03

- P3 : bon d'accord c'est mieux

- C : oui c'est

- P5 : ce qui manque un peu quand même [...] on ne parle pas si là on indique pas si on parle d'onde heu transmise ou heu

- P4 : ouais en fait c'était pour faire la relation entre les ondes transmises et puis la technique d'analyse

- P5 : et bein est-ce que tu peux pas rajouter

- P3 : et bein c'est là on rajoute deux colonnes là

- P5 : t'ajoutes encore deux colonnes

- P1 : ou une

- P3 : non tu mets onde transmise

- P1 : onde réfléchie transmise

- P3 : voilà

- P1 : et tu coches oui ou non

Le groupe aboutit finalement au tableau 3 ci-dessous, qui propose une entrée par les applications technologiques, qui sont à analyser du point de vue des phénomènes physiques qu'ils mobilisent.

\begin{tabular}{|c|c|c|c|}
\cline { 2 - 3 } \multicolumn{1}{c|}{} & \multicolumn{2}{c|}{ Émetteur/capteur placés } & \multicolumn{1}{c|}{ Onde analysée } \\
\cline { 2 - 4 } \multicolumn{1}{c|}{} & $\begin{array}{c}\text { du même côté du } \\
\text { corps à analyser }\end{array}$ & $\begin{array}{c}\text { de part et d'autre du } \\
\text { corps à analyser }\end{array}$ & $\square$ réfléchie $\square$ transmise \\
\hline Échographie & $\square$ & $\square$ & $\square$ réfléchie $\square$ transmise \\
\hline Radiologie & $\square$ & $\square$ & $\square$
\end{tabular}

Tableau 3 : Final

\section{Table 3: Final}

Cet extrait est assez typique des problèmes posés par le nouveau programme structuré par thème, en l'occurrence ici la santé, qui oblige à trouver des articulations parfois complexes ou artificielles entre la phénoménologie de la physique et des applications techniques. Le groupe mobilise ici de nombreux instruments pour modifier le tableau proposé par P4 : la prescription thématique du programme (I1), la phénoménologie de la physique (I2), un retour sur les difficultés de certains élèves 
face au tableau (I7); des connaissances sur des outils techniques (I8) et enfin la théorie spécifique des deux mondes (I4). Cette dernière joue ici un rôle implicite très important. En effet, lors des séances précédentes, la structure thématique du nouveau programme a conduit le groupe à de longs débats, éclairés par cette théorie, sur les croisements entre phénomènes et applications technologiques et la nécessité d'une clarification entre ces différents domaines de connaissance (en l'occurrence ici, des connaissances liées d'une part aux technologies et d'autre part à la physique) pour les élèves. C'est grâce à ce travail préalable que le groupe peut prendre des décisions.

L'action A4 (A4a/b) qui porte sur l'intérêt et la manière de formuler une définition d'une onde dans le modèle nous semble également intéressante du point de vue du rôle important joué par la théorie spécifique dans le processus de conception. Elle démarre par une proposition du chercheur du groupe (C) de légère modification du texte du modèle qui définit ce qu'est une onde (cf. Extrait 3).

Extrait 3 - 1:08:28

- C: alors y a une chose dans le modèle qui me gène [...] c'est pas grand-chose hein dans le modèle, mais je trouve que quand tu dis on appelle une onde la propagation d'un point à un autre d'une information l'onde ça contient l'information (2s) la propagation de l'information

La discussion se poursuit ensuite sur la signification physique de l'onde et la pertinence de la définition du modèle par rapport à la physique (I2). On se situe alors dans une phase de transposition d'une définition plus experte de l'onde vers une définition de la physique enseignée en seconde. Puis, suite à une remarque d'une enseignante (P1) l'échange s'oriente vers une prise en compte des connaissances initiales des élèves, liées au sens quotidien du mot « information» (I3) (cf. Extrait 4).

Extrait 4 - 1:10:14

- P3 : mais est-ce que ça vaut le coup de le mettre franchement

- P1 : bein si parce que ils vont se demander ce que c'est que cette information quoi hein

- A : quand même oui le mot information euh

- P1 : ils vont se dire tiens y a le journal Télé qui passe euh

- P2 : ouais l'information euh c'est

- P1 : qu'est-ce que ça veut dire pour eux l'information/

- P3 : ouais c'est je sais pas

Différents sens de la propagation de l'information sont alors évoqués : les informations à la télévision, l'information circulant sur Facebook, Twitter, etc. Les difficultés des élèves pour comprendre ce qu'est une onde sont aussi mises en avant, notamment à partir de l'expérience d'un enseignant ainsi que des tentatives de mise en place d'activités qui se révèlent peu compatibles avec les contraintes de temps (I7). Plus loin dans l'échange, un des enseignants finit par poser la question de l'utilité de cette définition qui finalement ne sert pas à grand-chose (cf. Extrait 5).

Extrait 5- 1:17:00

- P2 : mais bon fin bref enfin moi ce que je dis c'est que cette définition on se soulage la conscience en la donnant, mais

- P3 : pfff

- C : (rires)

- P2 : en même temps on n'en fait rien

- P4 : pratiquement rien

- P3 : en même temps on en fait rien et moi je vais même vous avouer j'ai carrément oublié pendant que j'étais dans des activités qu'elle était dans le modèle c'te définition c'est terrible

- P1 : ouais

- C : muhumm (2s)

- P3 : y a une élève qui m'a demandé monsieur à quoi elle sert cette définition putain j'étais mal quoi

Malgré des réticences que l'on peut sans doute relier à un élément de pratique enseignante assez répandu (on définit une notion avant de l'utiliser), le groupe décide finalement d'abandonner l'idée d'introduire une définition de l'onde dans le texte du modèle (A4b -1:29:44). 
Ce processus de décision s'appuie sur deux considérations. La première est liée à la théorie spécifique des deux mondes. Cette définition de l'onde n'est pas nécessaire pour comprendre et interpréter les concepts et notions en jeu dans les activités que les élèves réalisent. La cohérence entre les deux mondes est respectée en abandonnant cette définition. Ceci est confirmé dans la pratique par P3 dans l'extrait 5 ci-dessus. La deuxième considération est liée à la conformité aux compétences du programme officiel qui ne demande pas l'enseignement de cette définition (cf. Extrait 6) :

Extrait $6-1: 17: 15$

- P2: d'façon quand tu regardes dans les compétences attendues hein à aucun moment y a savoir définir une onde

- P3 : bien sûr de la même façon j'avais oublié que c'est en écrivant les compétences que je me suis rendu compte qu'il y avait ça

La théorie spécifique permet de prendre cette décision sans que les professeurs pensent que leur enseignement n'est pas fidèle à la physique. Ils n'ont pas besoin de « se soulager la conscience » comme le dit $\mathrm{P} 2$ puisqu'il y a cohérence entre les deux mondes, et que cette décision est compatible avec le programme officiel. La théorie spécifique joue ici contre un élément de la pratique professionnelle pas nécessairement toujours pertinent du point de vue de l'activité et de la compréhension des élèves

\section{Les limites de la théorie spécifique et des outils de conception}

A contrario, nous allons voir maintenant, à travers deux autres actions, les limites de l'appareillage théorique élaboré jusqu'ici, quand il s'agit de préciser des actions enseignantes lors de la mise en œuvre des séquences élaborées.

La première action $(\mathrm{A} 1 \mathrm{a} / \mathrm{b}$ - « Aider les élèves à cerner les savoirs importants à l'issue de la séquence ») est initiée par un des enseignants (P1) qui introduit un événement qui s'est produit le matin même dans sa classe à propos de la gestion par l'élève de ses brouillons (cf. Extrait 7). Les élèves attendent de l'institution (ici cela semble dépasser ce qui se passe avec ce seul professeur) que, dans leur cahier, seules des « choses » justes soient écrites, ce qui est à apprendre. P1 a accepté cette attente des élèves, même si ce n'est pas son attente a priori. Lors de la discussion qui suit P2 prolonge le débat sur la façon dont le professeur guide l'élève dans l'utilisation de son cahier lors des révisions ou du travail à la maison.

Extrait 7 - 1:01:50

- P1 : au bout du bout ce matin je leur ai filé euh des feuilles de brouillon quoi

- P3 : parce que, mais ça te gêne tant que ça que ils écrivent/

- P1 : non ça ne me gêne pas, mais c'est eux qui veulent pas

- P3 : ah bon

- P1 : soit ils prennent une belle feuille et ils écrivent trois trucs de brouillon, mais ils mélangent pas

- P3 : moi j'en ai quelques-uns qui font ça

- P1 : ils ont du mal heu même si on leur dit laisser les erreurs barrez les ne les effacez pas ils sont tout le temps en train d'effacer de mettre heu

- P2 : surtout que le contrat le contrat c'est quand on leur dit de réviser le chapitre 3 c'est de ne pas apprendre que le modèle c'est de

- P3 : ouais ouïs bien sûr

- P2 : d'avoir une maitrise de ce qui a été fait dans les activités or ça c'est un peu hypocrite des fois y a des activités j'ai envie de leur dire celle-là c'est pas la peine de la relire.

Ceci conduit à aborder la hiérarchisation des différents éléments du savoir enseigné. P3 réfère à sa pratique et confirme qu'il n'explicite pas cette hiérarchisation du fait de la contrainte de temps, mais qu'il faudrait le faire à la fin de chaque chapitre (cf. Extrait 8).

Extrait 8 - 1:02:35

- P3 : le problème c'est qu'on est pas non plus très clair parce que franchement je trouve qu'avec la contrainte de temps on met tout à plat au même niveau quoi [...] on a jamais le temps de se poser de 
prendre du recul [...] on devrait projeter le sommaire des activités et dire bon maintenant regardez qu'est-ce qu'on a fait là [...] pour le devoir qu'est-ce que vous auriez envie de réviser plutôt comme activité tu vois dire que toutes les activités sont pas identiques, homogènes

- P1 : c'est sûr

- P3 : ça je le fais jamais ça

- P2 : bein parce que t'as pas

- P3 : et jamais je le fais parce que ha ha (mime l'essoufflement)

- P2 : t'as jamais une demi-heure à consacrer à ça

- P3 : bein voilà j'ai jamais 10 minutes à consacrer à ça

Un peu plus tard, à la demande du chercheur (C), et alors que les compétences attendues sont projetées, la discussion ressurgit (cf. Extrait 9).

Extrait 9 - 1:34:50

- C: quand tu fais ça ça t'aide pas à revoir les titres des activités à dire ce qui est important ou pas/

- P3 : mais ça devrait, mais je le corrige pas

- C: ah voilà c'est ça

- P3 : c'est horrible

- C : d'accord

- P3 : c'est ça la catastrophe c'est qu'en fait je

- P1 : et ça t'arrive pas de temps en temps/parce que

- P3 : je regarde quand

- P1 : je... des fois je prends du temps par exemple moi ça je m’en sers quand ils font leur fiche mémo alors des fois ils sont complètement seuls ils le font pas

- P3 : je me dis toujours qu'il faudrait que je

- P1 : des fois j'arrive à trouver du temps

Cette action ne sera pas reprise par la suite et ne donne pas lieu à une proposition de mise sur le site d'un commentaire ou d'un document de formation pour les enseignants.

Lors des échanges, les concepteurs ont recours à quatre types d'instruments : une des caractéristiques des séquences SESAMES relative à la gestion des différents écrits des élèves (I5); la notion de contrat didactique utilisée par P2 pour caractériser ce qui est attendu des élèves par l'enseignant lors de ces séquences (I6), en accord avec les instructions officielles (I1); et enfin l'expérience de plusieurs concepteurs relative à ce point particulier (I7), en l'occurrence ici plus particulièrement celles de P1 qui initie l'échange puis celle de P3.

Pour bien comprendre la discussion, il faut savoir que le statut du cahier de l'élève a une histoire dans le groupe SESAMES lycée (Tiberghien, Vince, Gaidioz, \& Coince, 2011). Il est lié au contrat didactique et en particulier au statut de l'erreur. Plusieurs années auparavant, dans le cadre d'élaboration de séquences sur le son, les chercheurs du groupe avaient proposé et insisté pour que les élèves notent dans leur cahier, aussi bien les réponses aux activités réalisées en petits groupes que les corrections fournies par l'enseignant à l'issue de la phase de discussion. Leur raisonnement était que cela serait un signal fort pour les élèves de prise en considération de leurs propositions par l'enseignant. De plus, cela leur permettait, lors de la phase de correction, de ne pas seulement noter les bonnes réponses, mais aussi des explications sur le fait que certaines propositions n'étaient pas recevables du point de vue de la physique. Mais les enseignants étaient très réticents à cette idée, arguant du fait que, par expérience, les élèves ont beaucoup plus de mal à distinguer les bonnes réponses des mauvaises si rien ne les différencie dans leur cahier. Ce débat s'est longtemps poursuivi, stabilisé par des compromis tels que l'utilisation de couleurs différentes en fonction des statuts des écrits. Il a même donné lieu à la rédaction de ressources générales sur la conduite et la gestion de la classe lors de la mise en œuvre des activités proposées (ces ressources sont disponibles dans la rubrique « se former » du site pégase). Mais on voit à travers cet extrait que l'histoire n'est pas terminée et que des questions telles que : dans quelle mesure des propositions, calculs, ou autres écrits par l'élève au cours d'activités faites en petits groupes ou individuellement sont à garder dans son cahier? ; est-il pertinent qu'un élève relise ses erreurs quand il révise, retravaille à la maison par exemple? continuent 
de se poser. Elles sont le signe d'un élément probablement fort de la culture enseignante relative à la façon de gérer pratiquement l'erreur. C'est aussi un point sur lequel la contrainte de temps pèse de façon très forte. Ces deux éléments (décalage par rapport à la représentation que les enseignants ont de la culture de leurs pairs et temps) contribuent probablement à ce que le groupe n'essaie pas, une nouvelle fois pour certains membres, de normer la pratique sur ce point. Sur cet événement, la théorie des deux mondes ou les outils associés ne peuvent pas jouer. Il faudrait une autre théorie spécifique complémentaire qui serait centrée sur le pôle enseignement avec des liens vers les deux autres pôles et qui permettrait d'affirmer plus solidement certains choix face à des éléments de culture enseignante difficile à remettre en cause.

Une deuxième action lors de la séance porte sur le problème des activités ouvertes (A11 - «Comment le prof doit gérer l'activité 4 »). Elle démarre par une remarque de $\mathrm{P} 4$, l'enseignant récemment arrivé dans le groupe et se termine assez rapidement sur une réponse que lui fait P3 qui est celui qui a le plus d'ancienneté (cf. Extrait 10).
Extrait $10-1: 33: 33$
P4 : et là par rapport à cette activité parce que le fait qu'il y ait pas de traces écrites heu c'est quand même une activité assez importante en fait (inaudible) il y a l'aller et retour heu si ils trouvent rien c'est on vérifie on arrête là si ils trouvent quelque chose de différent c'est pas gênant c'est heu $\mathrm{P} 3$ : pff le prof gère
P4 : d'accord
P3 : moi je pense que c'est des activités où il faut qu'on joue le jeu de l'ouverture à ça n'empêche pas de faire des commentaires profs mais pff, mais c'est degré de liberté maximum
P4 : hum hum

Il s'agit ici d'un échange sur une activité qui n'est pas «standard » pour SESAMES (cf. annexe 2, activité 4) et le groupe a finalement peu d'outils pour aider l'enseignant à mettre en œuvre ce type d'activités plus ouvertes, sans demande explicite de trace écrite. Or on a vu la place occupée par cette trace dans les choix du groupe, y compris dans la théorie spécifique. L'activité de l'élève est ici beaucoup moins guidée qu'à l'habitude.

Cette expression « le prof gère », sous-entend finalement plusieurs choses :

- l'enseignant gère la trace écrite qu'il exige ou pas, c'est un problème de choix personnel dépendant de l'importance qu'il donne à cette activité et donc au thème, mais aussi en fonction de contraintes de temps;

- il gère aussi la validation ou pas de l'expérience faite par les élèves;

- enfin, il gère la cohérence expérimentale entre la mesure faite avec l'écho et la mesure faite au réglet...

Si l'on ajoute le fait qu'il n'y a pas de prescriptions bien précises dans le programme par rapport aux savoirs en jeu dans cette activité, il est finalement assez logique que le groupe ait peu de choses à dire, dans la mesure où une telle activité ouverte laisse beaucoup plus de place à l'action enseignante. Là encore, la théorie spécifique des deux mondes ne peut guère jouer de rôle dans la conception de ce type d'activité et pourrait être utilement complémentée par une théorie plus ancrée sur le pôle enseignant.

\section{3.- Éléments de réflexion pour une théorie intermédiaire orientant l'action enseignante}

Les extraits analysés précédemment mettent en évidence que les prises de décisions orientées vers la production semblent favorisées lorsque les outils de conception et la théorie spécifique des deux mondes peuvent jouer un rôle. La comparaison des deux dernières colonnes du tableau en annexe 3 conforte cette hypothèse puisque, à chaque fois que les concepteurs mobilisent ce type d'instru- 
ments (I4), l'action débouche sur un résultat.

Pour outiller davantage le processus de conception, il convient de donner des éléments qui contribuent à l'élaboration d'une seconde théorie spécifique, qui prenne beaucoup plus en compte le pôle enseignement de la figure 1 et débouche sur des outils d'aide à la conception d'une part, mais aussi par suite à la mise en œuvre des ressources produites.

\section{La TACD comme point de départ}

Nous devons en premier lieu faire un choix d'une ou plusieurs grande(s) théorie(s) relative(s) au pôle enseignement. Sur ce point, avant de continuer, il est important de faire une différence entre théories de l'enseignement et théories de l'action, de la pratique ou de l'activité enseignante. Une théorie de l'enseignement, comme la théorie des situations didactiques, prend en compte les savoirs et les élèves et part d'hypothèses sur ce qui peut favoriser l'apprentissage, en l'occurrence dans ce cas : la confrontation des élèves par l'action sur un milieu, qui va rétroagir et valider ou invalider des stratégies d'action porteuses de certains savoirs; un rôle de l'enseignant pour concevoir et dévoluer ce milieu, puis institutionnaliser les réponses, etc. Cette théorie a permis des ingénieries didactiques originales ${ }^{9}$. Par comparaison, les théories de l'activité, de l'action ou de la pratique enseignante sont tournées vers l'enseignant : elles ont pour finalité de comprendre son fonctionnement en tant qu'acteur professionnel dans des conditions situationnelles données, dont les élèves sont un élément parmi d'autres (Altet, 2002; Amigues, 2003; Goigoux, 2007; Ria, Serres, \& Leblanc, 2010). Ces théories ne prétendent pas donner d'éléments permettant d'orienter l'action enseignante, vers ce qui permet l'apprentissage des élèves. Les travaux qui les mobilisent permettent par contre de mettre en évidence des caractéristiques importantes des conditions et situations de l'exercice de la pratique enseignante et des logiques d'action et de pensée, construites par l'expérience au sein de ces situations.

Notre position est que la théorie spécifique à élaborer doit partir d'une ou plusieurs grandes théories de l'enseignement et de grandes théories issues d'un ou des deux autres pôles, tout en tenant fortement compte des résultats fournis par les recherches sur la pratique enseignante. Selon nous, ces travaux centrés sur l'enseignant doivent jouer un rôle similaire à l'expérience des enseignants utilisateurs des ressources SESAMES, mais complémentaire à ces feed-backs subjectifs car la pratique est ici analysée de façon objective et/ou avec des outils qui en décuplent le pouvoir analytique. Ils doivent permettre d'explorer et de tester les possibilités et impossibilités de mise en œuvre de certaines ressources compte tenu des caractéristiques des situations, des gestes professionnels et des contraintes culturelles du métier.

Dans cette perspective, la Théorie de l'Action Conjointe en Didactique (TACD) (Sensevy, 2007; Sensevy, Mercier \& Schubauer-Leoni, 2000) nous semble être un bon point de départ pour élaborer une théorie spécifique cohérente avec l'approche développée jusqu'ici. Construite à partir d'une reprise de concepts didactiques issus de la théorie des situations didactiques et d'autres issus de la théorie anthropologique de Chevallard, son objet est recentré sur l'action, mais d'une façon relativement différente des théories de la pratique enseignante : il s'agit d'une part de l'action didactique, c'està-dire de ces éléments de la praxis qui concernent plus directement les savoirs et leur dynamique dans la classe; et d'autre part d'une action structurellement envisagée comme conjointe, c'est-à-dire engageant l'enseignant et les élèves dans une relation ternaire avec des savoirs, fortement basée sur un processus de communication.

Dans les termes de la TACD, on peut considérer que le processus d'élaboration des ressources jusqu'à présent a consisté essentiellement à définir des actions (activités) orientées vers la modélisation (fonctionnement du savoir de la discipline enseignée) et des milieux de réalisation de celles-ci (textes des activités, objets et matériels à utiliser, modèles à mobiliser) qui fournissent des éléments de savoir.

9. De ce point de vue, la finalité de la théorie des situations didactiques est proche de celle d'une théorie pédagogique comme celle de Freinet ou d'autres, qui organise des conditions et situations particulières pour l'apprentissage. 
Ainsi, les choix d'apprentissage retenus (par exemple partir de ce que les élèves connaissent déjà ou peuvent comprendre) conduisent à des contraintes sur les milieux lors de conception par le groupe R\&D. Les actions A3 et A4 illustrent par exemple l'importance accordée au fait de concevoir des textes et donc des éléments du milieu, compréhensibles pour les élèves. Mais le rôle plus particulier de l'enseignant dans l'action conjointe n'a été que partiellement documenté (principalement par les essais et retours d'expérience individuels des enseignants-concepteurs). Ceci peut poser potentiellement problème dès que la part de l'enseignant dans l'action conjointe augmente comme on a pu le voir dans les actions A1 et A11. Si l'on prend l'exemple de la gestion des traces écrites relaté plus haut, à ce stade, seules des tentatives individuelles non théorisées sont menées au sein du groupe de concepteurs. Une façon de faire peut consister en des codes locaux avec les élèves : par exemple mettre une indication typographique spécifique pour des questions qui demandent une action de l'élève sans trace écrite; une autre pour les questions qui demandent d'écrire peu de choses sur la feuille d'énoncé elle-même et une autre encore pour les questions qui nécessitent une rédaction plus longue sur le cahier de l'élève.

De ce point de vue, les concepts apportés par la TACD, en particulier ceux de contrat didactique, de milieu, de chronogénèse, topogénèse et mésogénèse, sont intéressants pour penser ce que peut être la part spécifique de l'enseignant dans l'action conjointe.

\section{Fonctions assurées par une théorie spécifique sur deux actions étudiées}

On peut maintenant essayer de préciser la fonction que pourrait avoir la théorie spécifique à élaborer, à partir des exemples (actions A1 et A11) étudiés précédemment dans la partie 2.

Pour l'action A1, elle documenterait la gestion d'un contrat didactique mettant en jeu un même objet, le cahier de l'élève, mais dans des milieux institutionnels différents. La première situation, celle évoquée par P1, correspond à l'ici et maintenant de la classe. En effet, au cours de la réalisation d'une activité, la question est celle du support, cahier ou brouillon, sur lequel l'élève note ses réponses et leurs arguments, puis la correction. C'est souvent un contrat où le professeur donne ses préférences, par exemple s'il préfère que les élèves notent leurs réflexions sur du brouillon et réservent le cahier à la correction. Dans le cas de SESAMES, le contrat est guidé par un choix d'apprentissage : le rôle nécessaire de l'erreur dans la compréhension. La deuxième situation est celle de l'élève travaillant en dehors de la classe lorsqu'il fait ses devoirs à la maison ou quand il prépare une évaluation. Dans cette situation, des travaux (Coulaud, 2005; Johsua \& Félix, 2002) ont montré que les élèves faibles ou moyens n'avaient pas compris l'importance relative des différentes activités réalisées et informations données par le professeur. Une troisième situation est évoquée dans la discussion du groupe, il s'agit, pour le professeur d'expliciter l'importance des différents éléments de savoir introduits dans la classe. L'objectif est ici d'aider plus particulièrement les élèves faibles et moyens. La théorie spécifique à construire devrait permettre de rendre cohérents les contrats associés non seulement à ces trois situations, mais aussi à celle du devoir surveillé. Cette cohérence va aussi mettre en jeu la nature et le contenu de ces savoirs et la forme de leur mise en œuvre dans la classe.

Dans le cas de l'action A11 dont l'objet porte sur un type d'activité plus ouvert, la théorie spécifique à élaborer devrait outiller les concepteurs puis les utilisateurs sur la gestion de ce type d'activité où les élèves ne sont plus guidés par une suite de consignes écrites. Il conviendrait de donner des indications aux enseignants pour repérer, sans trop la perturber, l'activité des élèves afin d'estimer l'avancée du savoir (et du savoir-faire) en jeu (chronogénèse). Il faudrait aussi leur donner les moyens pour juger de la nécessité ou non, d'une intervention auprès des élèves à des moments clés nécessitant une aide (topogénèse) car le milieu (mésogénèse) ne fournit pas les rétroactions suffisantes pour assurer une autonomie des apprenants. Une telle théorie spécifique, combinée avec une prise en compte de l'épistémologie de la discipline et une analyse des connaissances initiales des élèves, peut s'appuyer sur le triplet des genèses comme outils de décomposition de ce type d'activité en sous-étapes de résolution, précisant des états de progression des savoirs, des rôles de chacun et des caractéristiques d'évolution du milieu. 


\section{Conclusion}

Cette étude constitue la première analyse menée sur l'activité de conception des séquences SESAMES et des ressources associées.

Elle a permis d'illustrer, sur plusieurs extraits, le rôle important de la théorie spécifique des deux mondes pour instrumenter et guider le processus concret de conception : lorsqu'elle peut être mobilisée, les actions permettent des débats allant dans le sens d'une plus grande explicitation des choix de conception et débouchent quasiment toujours sur une décision concrète. Il conviendrait, bien sûr, de conforter ce qui n'est encore qu'une hypothèse, par l'analyse d'autres extraits, notamment lors des deux réunions enregistrées qui n'ont pas encore été analysées. Cependant, ces premiers résultats nous semblent assez probants du point de vue de l'efficacité de la théorie spécifique et des outils associés. Mais ce que les membres du groupe R\&D SESAMES Physique au Lycée peuvent ressentir depuis quelques années quant aux limites de la théorie et des outils existants est aussi confirmé. Il manque des instruments aux concepteurs pour préciser le rôle des enseignants dans l'action conjointe, lorsque les séquences sont implémentées dans une classe.

L'analyse menée sur le processus de conception est relativement intéressante pour avancer dans le sens d'une théorie spécifique complémentaire de la première : les extraits étudiés fournissent de premières informations intéressantes sur l'intérêt de la TACD comme point de départ pour la mise au point et l'analyse de la fonction d'une théorie spécifique et des outils de conception associés, travail qui doit maintenant être poussé plus loin.

Nous voudrions conclure cet article par l'enjeu plus général de ce type de conception prenant en compte, par le biais de théories spécifiques, les différents pôles du système didactique (enseignement, apprentissage, savoirs). À l'heure où l'on peut constater un fort développement de recherches sur l'activité enseignante, il nous semble important de rappeler que la pratique enseignante dite experte, fruit d'une longue expérience sur le terrain, ne produit pas toujours ce qui est souhaitable en termes d'apprentissage pour les élèves. Le métier enseignant, pas plus que d'autres, ne fait pas exception sur cette question du développement de pratiques parfois inadéquates (Billett, 2001). Il $\mathrm{y}$ aurait donc un fort risque à ne prendre comme référence pour la conception de ressources enseignantes que des théories et travaux sur la pratique enseignante, un risque d'enfermement sur une culture métier que d'autres ont pu qualifier de risque de nécrose professionnelle (Clot, 2007). Or, on a pu constater combien cette logique de la pratique était forte et prégnante y compris parmi les enseignants du groupe $R \& D$, si aucun instrument solidement étayé ne venait la questionner, notamment du point de vue de ses effets sur la présentation du savoir aux élèves et leur apprentissage. Dans cette perspective, on situe l'enjeu de la construction de théories spécifiques et d'outils opératoires pour la conception collaborative entre enseignants et chercheurs. Ils peuvent contribuer à mieux articuler les savoirs issus de l'expérience des enseignants et ceux issus de recherches (sur les différents pôles du système didactiques), en fournissant des principes fondés théoriquement qui facilitent l'explicitation et guident les choix de conception, notamment dans le sens d'une plus forte prise en compte des spécificités des savoirs et des logiques d'apprentissage des élèves.

\section{RÉFÉRENCES}

Altet, M. (2002). Une démarche de recherche sur la pratique enseignante : l'analyse plurielle. Revue Française de Pédagogie, 138, 85-93.

Amigues, R. (2000). L'enseignement comme travail. In P. Bressous (Ed.), Les stratégies de l'enseignant en situation d'interaction (pp. 243-262). Paris: Rapport de synthèse pour le programme école et Sciences cognitives (ACI).

Amigues, R. (2003). Pour une approche ergonomique de l'activité enseignante. Skhole, Hors-série 1, 5-16. 
Bachelard, S. (1979). Quelques aspects historiques des notions de modèle et de justification des modèles. In P. Delattre \& M. Thellier (Eds.), Élaboration et justification des modèles (Vol. 1, pp. 3-19). Paris: Maloine S.A.

Billett, S. (2001). Learning in the Workplace: Strategies for Effective Practice. Crows Nest NSW: Allen \& Unwin Academic.

Bunge, M. (1973). Method and matter. Dordrecht, The Netherlands: Kluwer.

Chevallard, Y. (1991). La transposition didactique, du savoir savant au savoir enseigné. Grenoble: La Pensée Sauvage.

Chevallard, Y. (1992). Concepts fondamentaux de la didactique : perspectives apportées par une approche anthropologique du didactique. Recherche en didactique des mathématiques, 12(1), 73-112.

Clot, Y. (2007). De l'analyse des pratiques au développement des métiers. Éducation et didactique, 1(1), 8393.

Cobb, P., Confrey, J., diSessa, A., Lehrer, R., \& Schauble, L. (2003). Design experiments in educational research. Educational Researcher, 32(1), 9-13.

Coince, D., Miguet, A.-M., Perrey, S., Rondepierre, T., Tiberghien, A., \& Vince, J. (2008). Une introduction à la nature et au fonctionnement de la physique pour les élèves de seconde. Bulletin de l'Union des Professeurs de Physique et de Chimie, vol. 102, n ${ }^{\circ}$ 900, 3-20

Coulaud, M. (2005). Évaluer la compréhension des concepts de mécanique chez des élèves de seconde : Développement d'outils pour les enseignants. Thèse de doctorat, Université Lumière Lyon 2.

Darses, F. (2009). Résolution collective des problèmes de conception. Le Travail Humain, 72(1), 43-59.

Design-Based-Research-Collective. (2003). Design-based research: An emerging paradigm for educational inquiry. Educational researcher, 32(1), 5-8.

diSessa, A. (2006). A history of conceptual change research: threads and fault lines. In K. Sawyer (Ed.), Cambridge handbook of the learning sciences (pp. 265-282). Cambridge: Cambridge University Press.

Doudin, P.-A., Pfulg, L., Martin, D., \& Moreau, J. (2001). Entre renoncement et engagement. Un défi pour la formation continuée des enseignants. In C. Lafortune, C. Deaudelin, P.-A. Doudin, \& D. Martin (Eds.), $L a$ formation continue. De la réflexion à l'action. Québec: Presses de l'Université du Québec.

Duval, R. (1995). Sémiosis et pensée humaine, registres sémiotiques et apprentissage intellectuels [Semiosis and human thought, semiotic registers and intellectual learning]. Berne: Peter Lang.

Gaidioz, P., \& Tiberghien, A. (2003). Un outil d'enseignement privilégiant la modélisation. Bulletin de l'Union des Physiciens, 850, 71-83.

Gaidioz, P., Vince, J., \& Tiberghien, A. (2004). Aider les élèves à comprendre le fonctionnement de la physique et son articulation avec la vie quotidienne. Bulletin de l'Union des Physiciens, 866(1029-1042).

Giboin, A. (2004). La construction de référentiels communs dans le travail coopératif. In J.-M. Hoc \& F. Darses (Eds.), Psychologie ergonomique : tendances actuelles (pp. 119-139). Paris: PUF.

Giere, R. N. (1988). Explaining science: a cognitive approach. Chicago: University of Chicago Press.

Givry, D. (2003). Étude de l'évolution des idées des élèves de seconde durant une séquence d'enseignement sur les gaz. Thèse, Université Lumière Lyon 2, Lyon.

Goigoux, R. (2007). Un modèle d'analyse de l'activité des enseignants. Éducation et Didactique, 1(3), 47-70.

Hacking, I. (1983/2005). Representing and intervening. Cambridge: Cambridge University Press.

Jeannin, L., Veillard, L., \& Tiberghien, A. (2010). Appropriation de nouvelles ressources d'enseignement par des professeurs de physique-chimie en seconde. Recherches en didactique des sciences et des techniques, 1,267-292.

Johsua, S., \& Dupin, J.-J. (1993). Introduction à la didactique des sciences et des mathématiques. Paris: Presses universitaires de France. 
Johsua, S., \& Félix, C. (2002). Le travail des élèves à la maison : une analyse didactique en termes de milieu pour l'étude. Revue Française de Pédagogie, 141, 89-97.

Küçüközer, A. (2005). L'étude de l'évolution de la compréhension conceptuelle des élèves avec un enseignement. Cas de la mécanique en lère S. Thèse, Université Lumière Lyon 2, Lyon.

Le Marechal, J. F., Barbe, E., Roux, M., Jean-Marie, O., Roue, B., \& Vincent, D. (2004). Difficultés des élèves sur l'utilisation des ions dans le cadre des nouveaux programmes. Bulletin de l'union des professeurs de physique et de chimie, 98(867), 1399-1410.

Leontiev, A. N. (1979). The problem of activity in psychology. In J. V. Wertsch (Ed.), The concept of activity in soviet psychology. New York: M.E. Sharpe.

Mayen, P. (2005). Travail de relation de service, compétences et formation. In M. Cerf \& P. Falzon (Eds.), Travailler dans le service (pp. 59-81). Paris: PUF.

Meheut, M., \& Psillos, D. (2004). Editorial - Teaching-Learning sequences: aims and tools for science education research. International Journal of Science Éducation, 26(5), 515-535.

Psillos, D., \& Kariotoglou, P. (1999). Teaching fluids: intended knowledge and students. Actual conceptual evolution. International Journal of Science Education, 21(1), 17-38.

Ria, L., Serres, G., \& Leblanc, S. (2010). De l'observation vidéo à l'observation in situ du travail enseignant en milieu difficile : étude des effets sur des professeurs stagiaires. Revue Suisse des Sciences de l'Éducation, 32(1), 105-120.

Ruthven, K., Leach, J., Laborde, C., \& Tiberghien, A. (2009). Design Tools in Didactical Research: Instrumenting the Epistemological and Cognitive Aspects of the Design of Teaching Sequences. Educational Researcher, 38(5), 329-342.

Sensevy, G. (2007). Des catégories pour décrire et comprendre l'action didactique. In G. Sensevy \& A. Mercier (Eds.), Agir ensemble : Éléments de théorisation de l'action conjointe du professeur et des élèves (pp. 187-211). Rennes: Presses Universitaires de Rennes.

Sensevy, G., Mercier, A., \& Schubauer-Leoni, M. L. (2000). Vers un modèle de l'action didactique du professeur. A propos de la course à 20. Recherches en didactique des mathématiques, 20(3), 263-304.

Shulman, L. S. (1986). Those who understand: knowledge growth in teaching. Educational Researcher, 15(2), 4-14.

Spelke, E. S., Philips, A., \& Woodward, A. L. (1995). Infant's knowledge of object motion and human action. In D. Sperber, D. Premack \& A. Premack (Eds.), Causal cognition: a multidisciplinary debate (pp. 4478). Oxford: Clarenton Press.

Tiberghien, A., \& Malkoun, L. (2007). Différenciation des pratiques d'enseignement et acquisitions des élèves du point de vue du savoir. Éducation et Didactique, 1, 29-54.

Tiberghien, A., Vince, J., \& Gaidioz, P. (2009). Design-based research: Case of a teaching sequence on mechanics. International Journal of Science Éducation, 31(17), 2275-2314.

Tiberghien, A., Vince, J., Gaidioz, P., \& Coince, D. (2011). Professional development of teachers and researchers in a collaborative development of teaching resources. In C. Linder, L. Ostman, D. A. Roberts, P. O. Wickman, G. Erickson, \& A. MacKinnon (Eds.), Exploring the landscape of scientific literacy (pp. 255-271). New York: Routledge.

Vygotski, L. S. (1934/1997). Pensée et langage (3ème ed.). Paris: La Dispute.

Vygotsky, L. S. (1978). Mind in society: the development of higher psychogical processes. Cambridge: Harvard University Press.

Woolfolk Hoy, A., Davis, H., \& Pape, S. (2006). Teacher's knowledge and beliefs. In P. Alexander \& P. Winne (Eds.), Handbook of Educational Psychology (2nd ed., pp. 715-737). Mahwah: Lawrence Erlbaum.

\section{RÉSUMÉ}

Ce travail se situe en didactique des sciences, dans une perspective 
d'ingénierie de conception de ressources d'enseignement. Dans une première partie de l'article, nous rendons compte de la construction d'une théorie spécifique (théorie des deux mondes) et d'outils de conception associés, élaborés à partir de théories beaucoup plus générales sur le fonctionnement des savoirs et sur l'apprentissage dans le domaine de la physique et d'une prise en compte de l'expérience des enseignants. Cette théorie et ces outils servent à fonder théoriquement les choix concrets de conception de séquences d'enseignement de la physique-chimie au lycée. Dans une seconde partie, nous analysons l'activité collective de conception de ces séquences par un groupe R\&D, composé d'un chercheur et de cinq enseignants, pendant une réunion d'environ une heure. Le cadre théorique mobilisé est celui de la théorie de l'activité (Leontiev, 1979). L'analyse porte plus particulièrement sur le rôle de la théorie spécifique des deux mondes et des outils associés dans les actions collectives réalisées et leur aboutissement ou non en des décisions concrètes au sujet de la ressource produite. Nous montrons l'opérationnalité de cette théorie et de ces outils pour les actions orientées vers la conception d'activités et de milieux d'apprentissage, mais aussi leur limite quand il s'agit de guider l'action enseignante lors de la réalisation de ces séquences en classe. Dans une troisième partie, nous proposons les premiers éléments d'une théorie spécifique complémentaire à celle des deux mondes qui pourrait être construite à partir de la Théorie de l'Action Conjointe en Didactique et qui permettrait de fonder sur le plan théorique les actions enseignantes lors de la réalisation des séquences.

Mots CLÉS :

conception collective de ressources enseignantes ; didactique de la physique ; analyse de l'activité de ; théorie de l'activité.

\section{RÉFÉRENCEMENT}

Veillard, L., Tiberghien, A., \& Vince, J. (2011). Analyse d'une activité de conception collaborative de ressources pour l'enseignement de la physique et la formation des professeurs : le rôle de théories ou outils spécifiques. Activités, 8(2), pp. 202-227, http://www.activites.org/v8n2/v8n2.pdf

Article soumis le 29 avril 2011, accepté pour publication le 29 août 2011 


\section{Annexe 1-Extrait du programme de la classe de $2^{\text {nde }}\left(\right.$ BOEN spécial $n^{\circ} 4$ du 29 avril 2010)}

\section{LA SANTÉ}

Les citoyens doivent acquérir une culture scientifique de façon à procéder à des choix raisonnés en matière de santé. L'objectif de ce theme est de montrer et d'expliquer le rôle des sciences physiques et chimiques dans les domaines du diagnostic medical et des médicaments.

\section{NOTIONS ET CONTENUS}

\section{COMPETENCES ATTENDUES}

Le diagnostic médical : l'analyse de signaux périodiques, l'utilisation de l'imagerie et des analyses médicales permettent d'établir un diagnostic. Des exemples seront pris dans le domaine de la santé (électrocardiogramme, électroencéphalogramme, radiographie, échographie, fibroscopie, ...). L'observation de résultats d'analyses médicales permet d'introduire les notions de concentration et d'especes chimiques ainsi que des considérations sur la constitution et la structure de la matière.

\begin{tabular}{|c|c|}
\hline $\begin{array}{l}\text { Signaux périodiques: période, fréquence, tension } \\
\text { maximale, tension minimale. }\end{array}$ & $\begin{array}{l}\text { Connaitre et utiliser les définitions de la période et de } \\
\text { la fréquence d'un phénoméne périodique. } \\
\text { ldentifier le caractere periodique d'un signal sur une } \\
\text { durée domée. } \\
\text { Déterminer les caractéristiques d'un signal périodique. }\end{array}$ \\
\hline $\begin{array}{l}\text { Ondes sonores, ondes électromagnétiques. } \\
\text { Domaines de fréquences. }\end{array}$ & $\begin{array}{l}\text { Extraire et exploiter des informations concernant la } \\
\text { nature des ondes et leurs fréquences en fonction de } \\
\text { l'application médicale. } \\
\text { Connaitre une valeur approchee de la vitesse du son } \\
\text { dans l'air. }\end{array}$ \\
\hline $\begin{array}{l}\text { Propagation rectiligne de la lumiere. } \\
\text { Vitesse de la lumière dans le vide et dans l'air. }\end{array}$ & $\begin{array}{l}\text { Connaitre la valeur de la vitesse de la lumiére dans le } \\
\text { vide (ou dans l'air). }\end{array}$ \\
\hline Réfraction et réflexion totale. & $\begin{array}{l}\text { Pratiquer une demarche experrimentale sur la } \\
\text { réfraction et la réflexion totale. } \\
\text { Pratiquer une demarche experimentale pour } \\
\text { comprendre le principe de methodes d'exploration et } \\
\text { linfluence des proprietés des milieux de propagation. }\end{array}$ \\
\hline
\end{tabular}




\section{Annexe 2-Exemples d'activités et de modèle : cas des ondes sonores et électromagnétique}

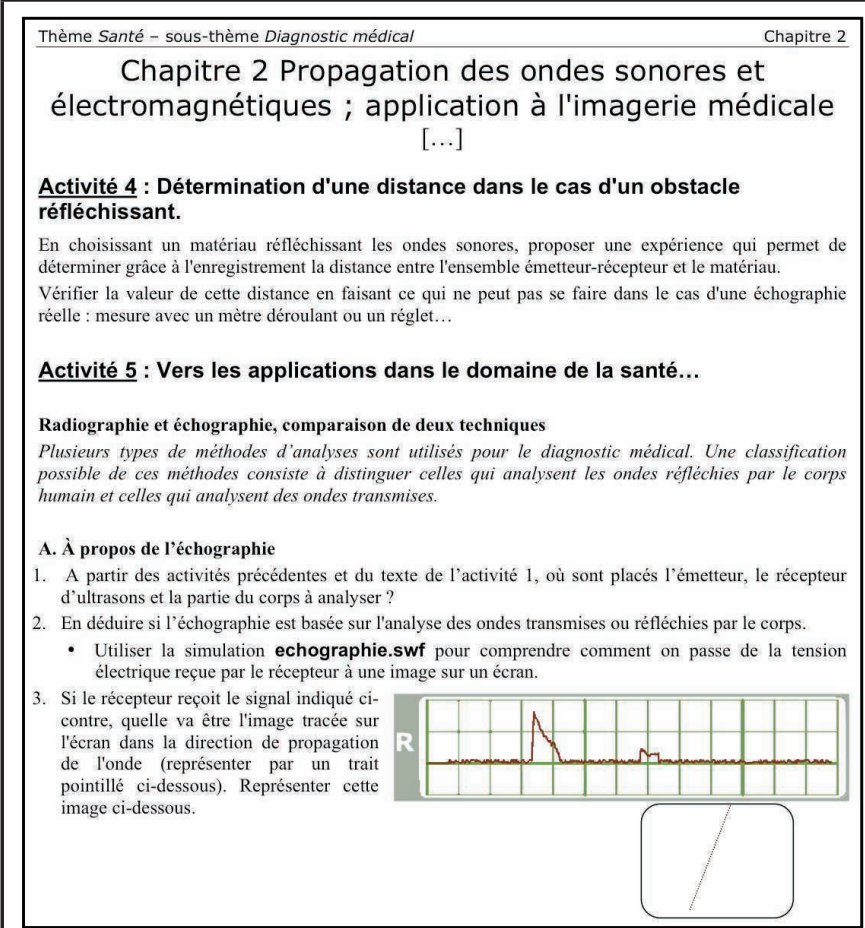

B. À propos de la radiographie

Le schéma de gauche ci-dessous illustre le principe de la radiographie. Le schéma de droite montre le résultat de la radiographie d'une main

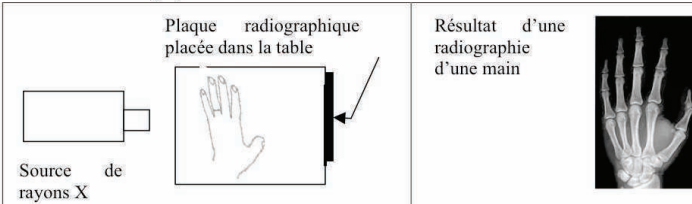

rayons $\mathrm{X}$

La plaque photographique utilisée, initialement transparente, noircit lorsqu'elle est atteinte par des rayons $\mathrm{X}$.

1- Représenter la chaine de propagation de l'onde à la manière d'une chaine sonore.

2- La radiographie est-elle basée sur l'analyse des ondes transmises ou réfléchies par le corps ?

3- A quel type d'ondes appartiennent les rayons utilisés? Justifier à l'aire du paragraphe $4 \mathrm{du}$ « modèle

des ondes sonores et électromagnétiques $)$

4- Sachant que les métaux réfléchissent ce type d'onde, indiquer comment va apparaitre une bague portéc au doigt sur la radiographie.

5- Quel est le domaine de fréquences des rayons $X$ ?

6- L'image de la radio de la main montre que les os et la chair de la main ont un effet différent sur les rayons $\mathrm{X}$. Expliquer précisément, en utilisant le vocabulaire du modèle quelle propriété distingue les os et la chair.

C. Conclusion

1 - Pour les deux premières colonnes, mettre une croix dans la bonne case.

2 - Cocher les bonnes réponses dans la colonne "Exemples".

\begin{tabular}{|c|c|c|c|}
\hline & \multicolumn{2}{|c|}{ Emetteur / capteur placés } & \multirow[b]{2}{*}{ exemple } \\
\hline & $\begin{array}{l}\text { du même coté du corps à } \\
\text { analyser }\end{array}$ & $\begin{array}{l}\text { de part et d' d'autre du corps } \\
\text { à analyser }\end{array}$ & \\
\hline Analyse de l'onde réfléchie & $\square$ & $\square$ & 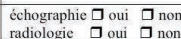 \\
\hline Analyse de l'onde transmise & $\square$ & $\square$ & $\begin{array}{l}\text { échographie } \square \text { oui } \square \text { non } \\
\text { radiologie } \square \text { oui } \square \text { non }\end{array}$ \\
\hline
\end{tabular}

Thème Santé - sous-thème Diagnostic médical $\quad$ Chapitre 2 Modèle des ondes sonores et électromagnétiques

1 - Définition d'une onde

On appelle " une onde » la propagation d'un point à un autre d'une information ou de la déformation d'un milieu sans que de la matière ne se soit déplacée entre ces deux points.

2 - Modèle des ondes sonores

L'« onde sonore » modélise, en physique, ce que nous appelons communément « le son 》.

Une onde sonore est la propagation de la vibration d'un milieu matériel

Les ondes sonores se propagent à la vitesse de $v=340 \mathrm{~m} / \mathrm{s}$ dans l'air, à $20^{\circ} \mathrm{C}$ et à pression atmosphérique.

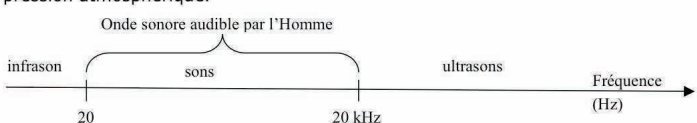

3 - Effet des milieux sur les ondes

Lorsqu'une onde arrive sur un obstacle, trois phénomènes peuvent intervenir : la transmission, la reflexion et I'absorption. Arrivant sur un obstacle, l'onde sonore ou I'obstacle) ou/et transmise (I'onde traverse l'obstacle) et/ou absorbée.
I'ong

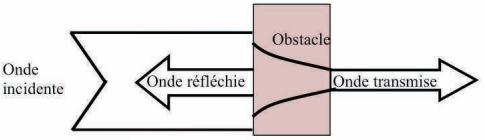

4- Modèle des ondes électromagnétiques

Un autre type d'ondes existent; ce sont les ondes électromagnétiques. Notre œil est sensible à certaines ondes électromagnétiques, dans un domaine de fréquence très restreint : c'est la lumière visible !

On peut classer les différentes ondes ́lectromagnétiaues selon leurs fréquences ou Oeurs longueurs d'onde comme nous l'avons vu en dit
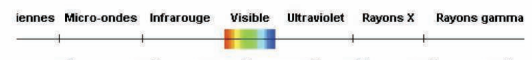
\begin{tabular}{lllllll}
$10^{9}$ & $10^{11}$ & $3,75.10^{14}$ & $7,50.10^{14}$ & $10^{17}$ & $10^{19}$ & $10^{22}$ \\
\hline
\end{tabular}

Longueu $\underset{3,10-1}{\longrightarrow} \longrightarrow_{(\mathrm{Hz})}$ d'onde Rappel $; 8,00.10^{-7} \mathrm{~m}=800 \mathrm{~nm}$ et $4,00.10^{-7} \mathrm{~m}=400 \mathrm{~nm}$ La vitesse de propagation des ondes électromagnétiques vaut dans le vide 


\section{Annexe 3 - Tableau synoptique des actions et instruments mobilisés}

(L'instrument I4, marqué en gras, correspond à la théorie spécifique des deux mondes et aux outils de conceptions déjà élaborés).

\begin{tabular}{|c|c|c|c|c|c|c|}
\hline Début & Durée & Séquence & $\mathbf{N}^{\circ}$ & Objet(s) - but de l'action & Instruments & $\begin{array}{l}\text { Actions donnant lieu à } \\
\text { production effective }\end{array}$ \\
\hline 1:01:50 & $2 \mathrm{~m} 10 \mathrm{~s}$ & Ondes & A1a & $\begin{array}{l}\text { Aider les élèves à cerner les savoirs importants } \\
\text { à l'issue de la séquence }\end{array}$ & I5 - I6 - I7 & \\
\hline 1:04:00 & $1 \mathrm{~m} 50 \mathrm{~s}$ & Ondes & A2 & $\begin{array}{l}\text { Situer les activités par rapport aux compé- } \\
\text { tences du programme }\end{array}$ & I1 - I5 - I7 & \\
\hline 1:05:29 & $2 \mathrm{~m} 59 \mathrm{~s}$ & Ondes & A3 & Simplifier un tableau trop compliqué & $\begin{array}{l}\text { I1 - I2 - I4 - I5 } \\
-\mathrm{I} 7\end{array}$ & Modification du tableau \\
\hline 1:08:28 & $11 \mathrm{~m} 25 \mathrm{~s}$ & Ondes & A4a & $\begin{array}{l}\text { Intérêt et termes d'une définition de l'onde } \\
\text { dans le modèle }\end{array}$ & I2 - I3 - I4 - I7 & \\
\hline 1:19:53 & $2 \mathrm{~m} 16 \mathrm{~s}$ & Ondes & A5 & $\begin{array}{l}\text { Préciser les différents types d'ondes sonores } \\
\text { dans le modèle }\end{array}$ & I2 - I3 - I4 & Modification schéma \\
\hline 1:22:09 & $4 \mathrm{~m} 48 \mathrm{~s}$ & Ondes & A6 & Intérêt et cohérence du schéma obstacle/milieu & $\begin{array}{l}\mathrm{I} 2 \text { - I3 - I4 - I5 } \\
-\mathrm{I} 7\end{array}$ & Maintien du schéma \\
\hline $1: 26: 35$ & $3 \mathrm{~m} 8 \mathrm{~s}$ & Ondes & A7 & $\begin{array}{l}\text { Modifier le schéma pour éviter que les élèves } \\
\text { pensent qu'une onde sonore peut contourner } \\
\text { un obstacle }\end{array}$ & $\begin{array}{l}\mathrm{I} 2-\mathrm{I} 33-\mathrm{I} 4-\mathrm{I} 5 \\
-\mathrm{I} 7\end{array}$ & Ajout propriétés onde \\
\hline $1: 29: 43$ & $8 \mathrm{~s}$ & Ondes & A4b & $\begin{array}{l}\text { Intérêt et termes d'une définition de l'onde } \\
\text { dans le modèle }\end{array}$ & & Suppression du modèle \\
\hline 1:29:52 & $1 \mathrm{~m} 33 \mathrm{~s}$ & Ondes & A8 & $\begin{array}{l}\begin{array}{l}\text { Réorganiser les différentes parties du modèle } \\
\text { pour assurer la cohérence }\end{array} \\
\end{array}$ & I4 - I5 - I7 & $\begin{array}{c}\text { Modification de l'ordre } \\
\text { des activités }\end{array}$ \\
\hline $1: 31: 25$ & $1 \mathrm{~m} 22 \mathrm{~s}$ & Ondes & A9 & $\begin{array}{l}\text { Intérêt de la partie sur la longueur d'onde dans } \\
\text { le modèle }\end{array}$ & I2 - I4 - I5 - I7 & Ajout énergie \\
\hline $1: 32: 48$ & $45 \mathrm{~s}$ & Ondes & A10 & $\begin{array}{l}\begin{array}{l}\text { Explication des modifications réalisées sur le } \\
\text { schéma de l'échographie }\end{array} \\
\end{array}$ & I5 - I7 & \\
\hline 1:33:33 & $1 \mathrm{~m} 14 \mathrm{~s}$ & Ondes & A11 & Comment le prof doit gérer l'activité 4 ? & I1 - I5 & \\
\hline $1: 34: 47$ & $26 \mathrm{~s}$ & Ondes & A1b & $\begin{array}{l}\text { Aider les élèves à cerner les savoirs importants } \\
\text { à l'issue de la séquence }\end{array}$ & I1 - I2 - I5 - I7 & \\
\hline 1:35:14 & $19 \mathrm{~s}$ & Ondes & A12 & Échanger sur le planning d'enseignement & I1 - I5 - I7 & \\
\hline 1:35:33 & $37 \mathrm{~s}$ & Ondes & A13a & Mettre la séquence sur Pégase & $\mathrm{I} 5$ & \\
\hline $1: 36: 10$ & $1 \mathrm{~m} 50 \mathrm{~s}$ & Ondes & A14 & $\begin{array}{l}\text { Explication de la mise en œuvre de la fontaine } \\
\text { lumineuse }\end{array}$ & I2 - I7 & \\
\hline 1:38:00 & $1 \mathrm{~m} 35 \mathrm{~s}$ & Ondes & A13b & Mettre la séquence sur Pégase & I5 & \\
\hline $1: 39: 36$ & $37 \mathrm{~s}$ & Ondes & A15a & Le train du sommeil & \begin{tabular}{|l|}
$\mathrm{I} 1$ - I2 - I4 - I5 \\
$-\mathrm{I} 7$
\end{tabular} & \\
\hline 1:40:14 & $44 \mathrm{~s}$ & Ondes & A16 & $\begin{array}{l}\text { Explication du fonctionnement des éditeurs } \\
\text { (P3) }\end{array}$ & I4 - I8 & \\
\hline 1:40:58 & $2 \mathrm{~m} 22 \mathrm{~s}$ & Ondes & A13c & Mettre la séquence sur Pégase & I5 & \\
\hline 1:43:21 & $7 \mathrm{~s}$ & Ondes & A15b & Intérêt de l'activité sur le train du sommeil & & $\begin{array}{l}\text { Basculement en exer- } \\
\text { cice }\end{array}$ \\
\hline 1:43:28 & $3 \mathrm{mn}$ & Ondes & A13d & Mettre la séquence sur Pégase & $\mathrm{I} 5$ & \\
\hline 1:46:28 & $1 \mathrm{~m}$ & Ondes & A17 & $\begin{array}{l}\text { Résoudre le problème de la bague manquante } \\
\text { sur la photo }\end{array}$ & $\mathrm{I} 2$ - I3 - I4 - I5 & $\begin{array}{l}\text { Question supplémen- } \\
\text { taire }\end{array}$ \\
\hline 1:47:40 & $48 \mathrm{~s}$ & Optique & A18 & $\begin{array}{l}\text { Rédaction du doc prof partie optique : qui et } \\
\text { quand? }\end{array}$ & I5 - I7 & \\
\hline 1:48:27 & $5 \mathrm{~m} 23 \mathrm{~s}$ & Mécanique & A19 & Explication par des activités faites par P1 & I1 - I5 - I7 - I8 & \\
\hline 1:53:51 & $2 \mathrm{~m} 15 \mathrm{~s}$ & Toutes & A20 & $\begin{array}{l}\text { Trouver des liens avec les thèmes : faire les } \\
\text { thèmes ou pas? }\end{array}$ & $\mathrm{I} 1$ - I5 - I7 & \\
\hline 1:56:06 & $2 \mathrm{~m} 27 \mathrm{~s}$ & Mécanique & A21 & $\begin{array}{l}\text { De l'intérêt de faire taper dans un ballon et } \\
\text { prendre des mesures }\end{array}$ & $\mathrm{I} 2-\mathrm{I} 3-\mathrm{I} 7$ & \\
\hline 2:01:20 & $1 \mathrm{~m} 35 \mathrm{~s}$ & NA & A22 & Fixer les prochaines dates de réunion & 1 & \\
\hline 2:02:56 & $1 \mathrm{~m} 02 \mathrm{~s}$ & Mécanique & A23 & Quel lien avec le thème sport? & $\mathrm{I} 1-\mathrm{I} 2$ - I5 - I7 & \\
\hline
\end{tabular}

